\title{
The effect of maternal omega-3 fatty acid supplementation on behaviour and kindling rates of seizure-prone and seizure-resistant offspring
}

\author{
by \\ Marissa Kelso \\ A thesis submitted to \\ the Faculty of Graduate and Postdoctoral Affairs \\ in partial fulfillment of the requirements for the degree of \\ Master of Science \\ in
}

Psychology specialization in Neuroscience

Carleton University

Ottawa, Canada

C2010 Marissa Kelso 
Library and Archives

Canada

Published Heritage

Branch

395 Wellington Street

Ottawa ON K1A ON4

Canada
Bibliothèque et

Archives Canada

Direction du

Patrimoine de l'édition

395 , rue Wellington

Ottawa ON K1A ON4

Canada
Your file Votre référence

ISBN: 978-0-494-71586-4

Our file Notre référence

ISBN: 978-0-494-71586-4
NOTICE:

The author has granted a nonexclusive license allowing Library and Archives Canada to reproduce, publish, archive, preserve, conserve, communicate to the public by telecommunication or on the Internet, loan, distribute and sell theses worldwide, for commercial or noncommercial purposes, in microform, paper, electronic and/or any other formats.

The author retains copyright ownership and moral rights in this thesis. Neither the thesis nor substantial extracts from it may be printed or otherwise reproduced without the author's permission.
AVIS:

L'auteur a accordé une licence non exclusive permettant à la Bibliothèque et Archives Canada de reproduire, publier, archiver, sauvegarder, conserver, transmettre au public par télécommunication ou par l'Internet, prêter, distribuer et vendre des thèses partout dans le monde, à des fins commerciales ou autres, sur support microforme, papier, électronique et/ou autres formats.

L'auteur conserve la propriété du droit d'auteur et des droits moraux qui protège cette thèse. $\mathrm{Ni}$ la thèse ni des extraits substantiels de celle-ci ne doivent être imprimés ou autrement reproduits sans son autorisation.
In compliance with the Canadian Privacy Act some supporting forms may have been removed from this thesis.

While these forms may be included in the document page count, their removal does not represent any loss of content from the thesis.
Conformément à la loi canadienne sur la protection de la vie privée, quelques formulaires secondaires ont été enlevés de cette thèse.

Bien que ces formulaires aient inclus dans la pagination, il n'y aura aucun contenu manquant.

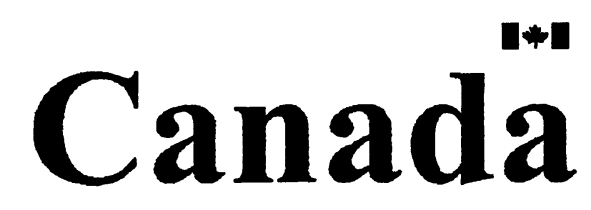




\begin{abstract}
Optimal maternal omega-3 levels during gestation and infancy are important for proper offspring neurodevelopment as deficits have been linked to disorders such as ADHD/ASD. This relationship is modeled in selectively bred seizure-prone (FAST) and seizure-resistant (SLOW) rat strains. The present study examined the effect of maternal omega-3 supplementation on behaviour and kindling rates of FAST and SLOW offspring. Results indicate supplementation significantly increased memory in FAST rats while reducing impulsivity in FAST rats and kindling rates in SLOW rats. Of concern, supplementation significantly reduced pup viability in FAST rats, and lead to a reduction in blood plasma levels of omega- 3 fatty acids in both strains. This negative effect on pup viability indicates omega-3 supplementation, in individuals predisposed to disorders such as $\mathrm{SD} / \mathrm{ADHD} / \mathrm{ASD}$, may result in irregular neurodevelopment which is fatal and, thus, caution must be taken when considering supplementation during pregnancy.
\end{abstract}




\section{Acknowledgments}

I am grateful to everyone who has helped me along my journey at Carleton. To begin, I would like to thank my parents for giving me many opportunities necessary for my success. The wonderful support I have received throughout the past two years has made it possible for me to reach this goal. Thank you also to my Aunt Carol, and cousins Kyler and Karina who have been so generous and made my time here in Ottawa better than I could have imagined.

I am indebted to both Dan McIntyre and Krista Gilby for their ongoing guidance and support during my graduate training at Carleton. Without their knowledge and understanding I would not have been able to achieve my goals. To my lab mates, Dwayne, Véro and Mike, I appreciate your aid over the past two years which has greatly contributed to my learning. A special thanks to Etelle Bourassa for her compassion and positive support as well as to Alfonzo Abizaid for opening a new door in my life by granting me access to neuroscience at Carleton.

Learning is a treasure that will follow its owner everywhere. -Chinese Proverb 


\section{Table of Contents}

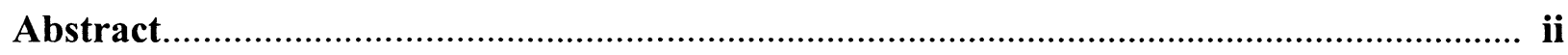

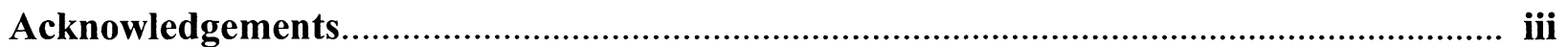

List of Abbreviations...................................................................................................... vi

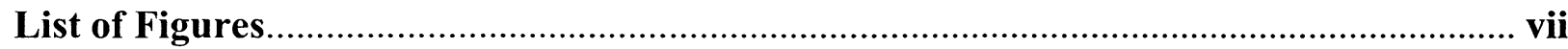

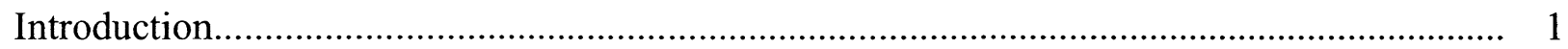

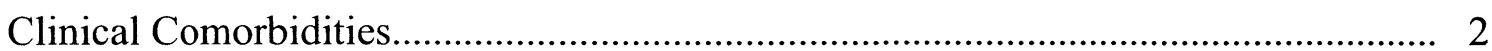

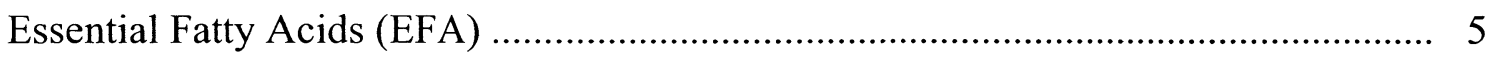

Essential Fatty Acid Supplementation..................................................................... 8

Maternal Omega-3 Fatty Acid Supplementation............................................................ 10

Seizure-prone versus Seizure-resistant Rat Strains.................................................... 12

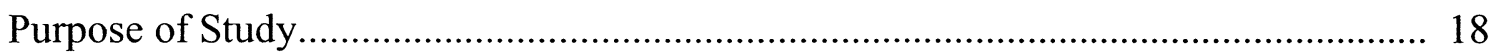

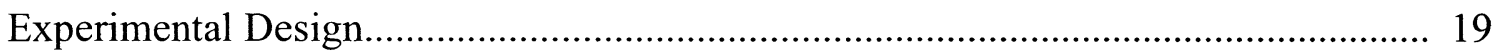

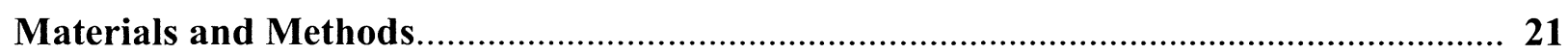

Animals and Treatment................................................................................ 21

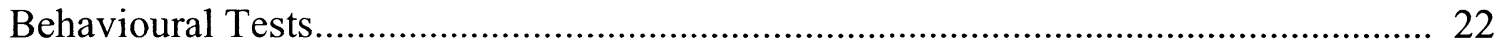

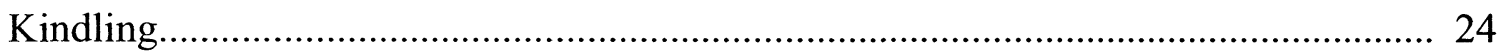

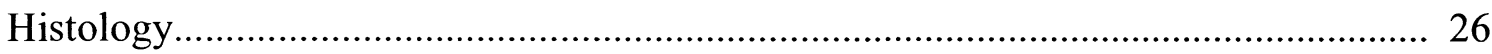

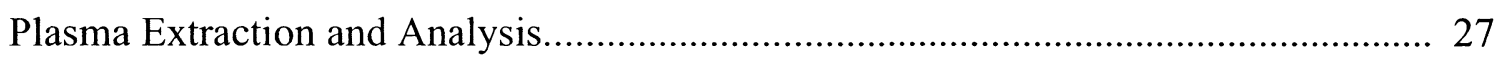

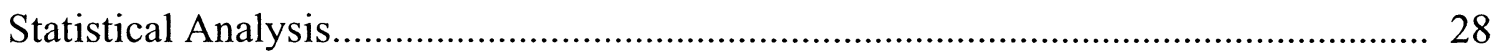

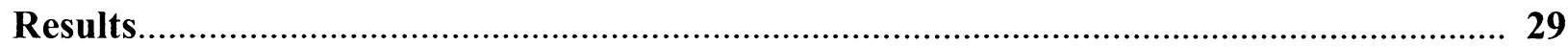

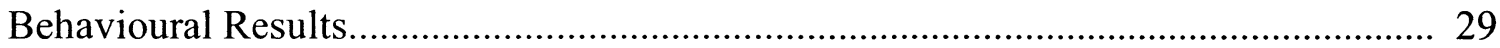

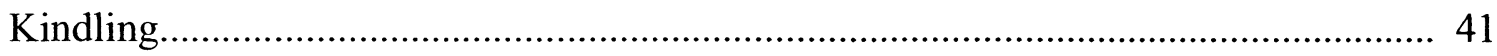

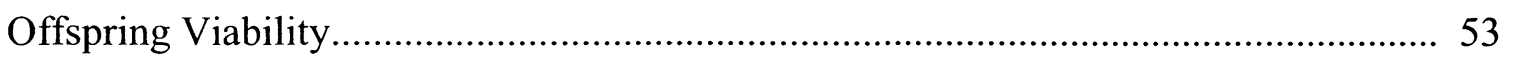

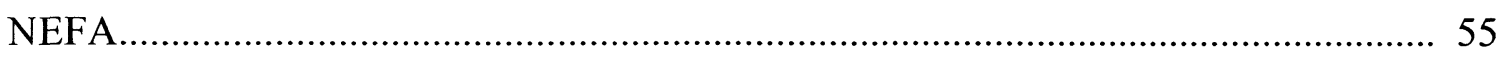

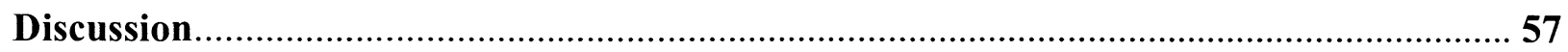

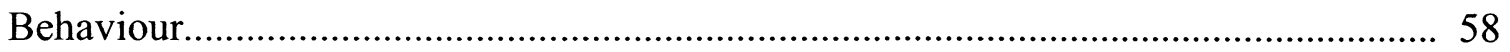




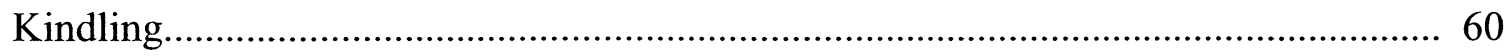

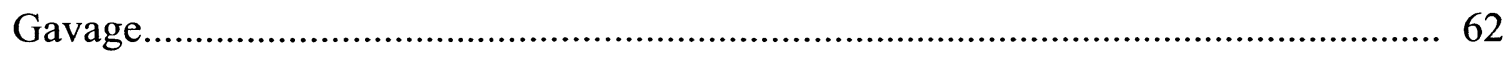

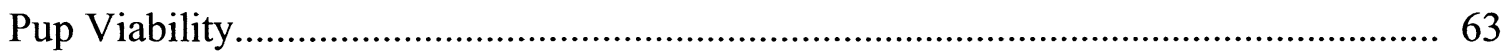

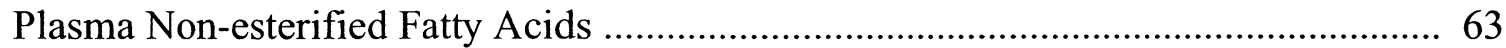

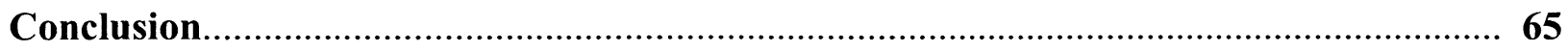

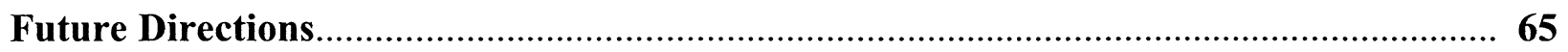

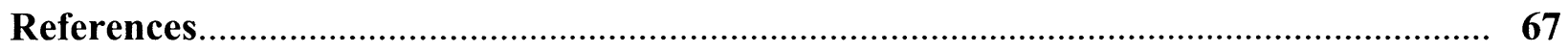




\section{LIST OF ABBREVIATIONS}

\begin{tabular}{|c|c|}
\hline ADHD & Attention Deficit Hyperactivity Disorder \\
\hline $\mathrm{ASD}$ & Autistic Spectrum Disorder \\
\hline $\mathrm{SD}$ & Seizure Disorder \\
\hline DHA & Docosahexanoic Acid \\
\hline EFA & Essential Fatty Acid \\
\hline EPA & Eicosapentanoic Acid \\
\hline FA & Fatty Acids \\
\hline $\mathrm{FC}$ & Fast Control Group \\
\hline FC-G & Fast Control Gavaged Group \\
\hline FTx & Fast Treatment Group \\
\hline KR & Kindling Rate \\
\hline MWM & Morris Water Maze \\
\hline NDD & Neurodevelopmental Disorders \\
\hline NEFA & Non-esterified Fatty Acids \\
\hline $\mathrm{OF}$ & Open Field \\
\hline PUFA & Polyunsaturated Fatty Acids \\
\hline $\mathrm{R}$ & Restraint \\
\hline $\mathrm{SC}$ & Slow Control Group \\
\hline $\mathrm{SC}-\mathrm{G}$ & Slow Control Gavaged Group \\
\hline STx & Slow Treatment Group \\
\hline
\end{tabular}




\section{LIST OF FIGURES}

Figure 1: Omega-3 and omega-6 fatty acid breakdown

Figure 2: Relative activity levels in the open field paradigm

Figure 3: Relative learning and memory levels in the MWM

Figure 4: Relative learning and memory levels on Day 1 in the MWM

Figure 5: Relative learning and memory levels on Day 2 in the MWM

Figure 6: Relative learning and memory levels on Day 3 in the MWM

Figure 7: Relative learning and memory levels on Day 4 in the MWM

Figure 8: Relative swimming abilities in the MWM swim test

Figure 9: Relative hyperactivity/impulsivity in the restraint test

Figure 10: Mean pre-kindled ADT associated with local amygdala excitability

Figure 11: Mean pre-kindled ADD associated with local amygdala excitability

Figure 12: Mean kindling rate to the first stage-5 seizure

Figure 13: Mean cumulative ADD to the first stage-5 seizure

Figure 14: Mean latency to onset of partial seizures (stage 1-4) associated with elicitation of 6 stage-5 seizures

Figure 15: Mean duration of partial seizure (stage 1-4) behaviour associated with elicitation of 6 stage-5 seizures

Figure 16: Mean duration of fully generalized seizure behaviour associated with elicitation of 6 successive stage-5 seizures

Figure 17: Mean ADD associated with elicitation of 6 successive stage-5 seizures

Figure 18: Mean offspring viability rates

Figure 19: Mean plasma NEFA levels 


\section{Introduction}

Over the last thirty years, science has begun to methodically examine the aetiology of neurodevelopmental disorders (NDD). These disorders typically involve symptoms that arise out of impaired growth and development of the central nervous system (CNS) resulting from genetic, immune, metabolic and/or nutritional causes (Richardson, 2006; Richardson \& Ross, 2000). NDDs such as Attention Deficit Hyperactivity Disorder (ADHD) and Autistic Spectrum Disorders (ASD) have come to be of grave concern in North America due to their escalating rates of diagnosis over the past decade, as well as the economic and social challenges they present.

ADHD was first reported in 1955 by Ounstead, who used the term "hyperkinetic syndrome" to describe the disorder. According to the DSM-IV (American Psychological Association, 2000), ADHD is characterized by a persistent pattern of inattention, impulsivity and/or hyperactivity that is present for at least six consecutive months prior to the age of seven, and in more than one setting. Although ADHD is one of the most common childhood and adolescent disorders, it remains difficult to diagnose due to the large number of associated symptoms that can appear in an array of combinations. It has been speculated that ADHD affects between $3-5 \%$ of school-age children, however, estimated rates increase to $10 \%$ when diagnostic criteria are less stringent (Biederman \& Faraone, 2005).

Recently, ADHD has been classified as part of the ASD, a group of disorders defined by symptoms such as impaired social interaction and communication as well as restricted and repetitive behaviours (American Psychological Association, 2000). Autism, Asperger's syndrome and Pervasive Developmental Disorder Not Otherwise Specified (PDD-NOS) are all 
included under the rubric of ASD, and together this collection of disorders, excluding those with ADHD alone, reportedly affects between $0.2-0.6 \%$ of the population (Gabis, Pomeroy, \& Andriola, 2005). Research has also shown both ADHD and ASD are hereditary, however, rates vary greatly with ADHD heritability reported to be between 0.39 and 0.91 (Faraone \& Biederman, 1994; Ronald et al., 2006) and ASD heritability between 0.64 and 0.92 (Ronald et al., 2006). It is likely that these variations are associated with the diagnostic inconsistencies mentioned earlier.

\section{$\underline{\text { Clinical Comorbidities }}$}

Although at this time the cause(s) responsible for the manifestation of NDDs such as ADHD/ASD are not fully understood, the high clinical co-diagnosis of these disorders and epilepsies suggests that they may have a common underlying cause. For example, despite ADHD affecting about $3-7 \%$ of the general pediatric population, the rate of diagnosis in children suffering from epilepsy is about 2.5 fold greater (Hesdorffer et al., 2004; Kaufmann, GoldbergStern, \& Shuper, 2009; Sherman, Slick, Connolly, \& Eyrl, 2007). Furthermore, the reverse relationship has been observed in children diagnosed with ADHD, where rates of epilepsy diagnosis are 2.7 times greater than in otherwise normal healthy individuals (1\%) (Davis et al., 2010). ASD has also been shown to be highly comorbid with epilepsy with up to $40 \%$ of individuals diagnosed with ASD (not including ADHD, 0.2-0.6\%) also being epileptic (Gabis, et al., 2005).

Secondary research investigating multiple diagnoses of ADHD/ASD and seizure disorders (SD) suggests that epilepsy is more severe (earlier seizure onset, more frequent seizures) in children diagnosed with ADHD than in those who have not been diagnosed with 
ADHD (Davis, et al., 2010). This increase in severity of behavioural symptoms is likely related to greater electroencephalographic abnormalities (5.6-30.1\%) in children with multiple diagnoses (e.g., ASD and epilepsy) compared to healthy children, or those with a single diagnosis (3.5\%) (Kaufmann, et al., 2009).

The precise neurological mechanisms involved in a predisposition towards and/or clinical expression of $\mathrm{SD} / \mathrm{ADHD} / \mathrm{ASD}$ remain unknown, however, documented high co-morbidity between these disorders is most likely due to one of three reasons (Kaufmann, et al., 2009). First, an independent circumstantial relationship may exist; all of these disorders affect young individuals at a high rate and, thus, a high rate of natural overlap might be expected. Second, there may be a direct causal relationship between these disorders, where epileptic brain activity results in, or aggravates, ADHD/ASD symptoms, or vice-versa. Finally, there may be an underlying causal mechanism that is ultimately responsible for the co-manifestation of these disorders. Potential mechanisms might include genetic or environmental factors or an interaction between environmental and genetic/epigenetic factors. This final possibility is of particular interest given the link between fatty acid (FA) deficiencies and ADHD/ASD and epilepsy (Young, Gean, Chiou, \& Shen, 2000), and the high heritability rates of ADHD (39-91\%)/ASD (64-92\%) (Ronald, et al., 2006) and epilepsy (80\%) (Kieldsen et al., 2003).

Previous investigations have explored neuroanatomical and neurophysiological mechanisms as well as genetics as possible areas responsible for the manifestation of these disorders (Ballard et al., 1997). The observed association between increasing rates of $\mathrm{ADHD} / \mathrm{ASD}$ diagnosis and the increase in consumption of foods high in fat and low in nutritional value within North America, however, has also led to scientific speculation that 
nutrition may play a causal role (Ottoboni \& Ottoboni, 2003; Simopoulos, 2001). The success of the ketogenic diet (high fat, low carbohydrate/protein) in reducing seizure prevalence in both humans and animals, as well as improving behavioural symptoms exhibited by individuals diagnosed with ADHD (MacCraken \& Scalisi, 1999; Pulliainen et al., 2000), further indicates these disorders may be related based on FA metabolism irregularities (Agale, Kulkarni, Ranjekar, \& Joshi, 2010; Freeman \& Vining, 1999; Freeman et al., 1998; Gilby, Crino, \& McIntyre, 2007; Murphy, Likhodii, Hatamian, \& McIntyre Burnham, 2005; Wainwright, 2002; Yuen \& Sander, 2004). Indeed, continued research has indicated FA deficiencies are associated with the development of ADHD/ASD and SD. Specifically, individuals diagnosed with ADHD/ASD show reduced plasma levels of essential FAs (EFA) compared to healthy controls (Chalon, 2009; Johnson, Ostlund, Fransson, Kadesjo, \& Gillberg, 2009; Raz \& Gabis, 2009). While a precise FA deficiency has not been linked with the development of SD, research (discussed below) has indicated omega-3 supplementation leads to a decrease in severity of symptoms and promotes neuroprotection (Cysneiros et al., 2009), thus indicating EFAs may play a role in seizure disorders (Scorza et al., 2008; Tavriger, 1966; Yuen \& Sander, 2004; Yuen et al., 2005).

An in-depth investigation into the role of EFAs in central nervous system functioning will contribute to the understanding of how EFAs might be influencing the onset of SD and NDDs such as ADHD/ASD, how symptomology associated with these disorders have been affected by omega-3 supplementation throughout child- and adulthood, and introduce a possible treatment that may prevent disorder manifestations altogether. 


\section{Essential Fatty Acids (EFAs)}

EFAs are considered essential as they must be ingested through a dietary source due to the body's inability to synthesize this complex group of molecules. They have been classified into two categories: omega-3 (n-3) and omega-6 (n-6), which differ chemically according to the location of the first double bond in the molecule. The primary omega-3 FA (ALA, 18:3(n-3)) and omega-6 FA (LA, 18:2(n-6)) precursors are molecules that serve as a starting compound for the synthesis of longer polyunsaturated FAs (PUFAs), including eicosapentaenoic acid (EPA, 20:5(n-3)), docosahexanoic acid (DHA, 22:6(n-3)) and arachidonic acid (AA, 20:4(n-6)) (see Figure 1). FA precursors (ALA, LA) are typically found in plants (flax seed, walnuts), while PUFAs can be synthesized from these precursors or consumed directly from more complex organisms such as fatty fish (salmon, tuna, and mackerel) (Cetin, Alvino, \& Cardellicchio, 2009).

DHA is an essential omega-3 PUFA and is associated with normal growth and functioning of nervous tissue (Genuis \& Schwalfenberg, 2006; Helland, Smith, Saarem, Saugstad, \& Drevon, 2003). Optimal DHA levels during development of the fetal and infant brain are essential for proper neurogenesis and neurotransmission, and thus dictate cognitive development, visual acuity, hand eye coordination and attention span (Innis, 2007). In contrast, deficiencies are associated with reduced cognitive and behavioural performance resulting from aberrant neurodevelopment in areas such as neuronal arborisation and myelin synthesis (ClarkTaylor \& Clark-Taylor, 2004; Raz \& Gabis, 2009). Indeed, FA deficiencies throughout pregnancy and lactation have been shown to delay myelin growth and maturation in the frontal lobes in offspring (Saugstad, 2004). Moreover, human research has demonstrated 
LA (linoleic acid) Omega 6

Vegetable oils, seeds, nuts

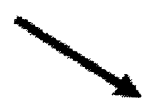

ALA, alpha linolenic acid Omega 3

flaxseed oil, walnuts, flaxseed, canola oil

Delta 6 desaturase

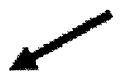

Gamma -linoleic acid (GLA: borage \& primrose oil)

$\downarrow$

Arachidonic Acid (meat, eggs, dairy) $\downarrow \neq$ NSAIDs

Pro-inflammatory Omega 6 Eicosanoids
EPA (fish)

$\checkmark$

DHA (fish)

$\downarrow$

Omega 3 Eicosanoids

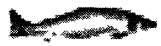


neurocognitive deficits as a consequence of reduced DHA levels during development may manifest as deficits in attention and an increased risk for ADHD (Brookes, Chen, Xu, Taylor, \& Asherson, 2006; Raz \& Gabis, 2009), schizophrenia (Chalon, 2009; McNamara \& Carlson, 2006) and certain types of epilepsy (Young, et al., 2000). Similarly, rats with reduced omega-3 FA levels show hyperactivity and reduced memory and learning compared to healthy controls (Agale, et al., 2010; Gilby, Crino, et al., 2007; Murphy, et al., 2005; Wainwright, 2002), further indicating optimal DHA levels are necessary for proper neurodevelopment. 


\section{$\underline{\text { Essential Fatty Acid Supplementation }}$}

Based on the reported association between EFA deficits and neurodevelopemental disorders, researchers have investigated the possibility of treating SD and ADHD/ASD through supplementation of omega-3 FAs. Results of these investigations have been conflicting with some studies indicating a positive effect of FA supplementation (Ferrari et al., 2008; Johnson, et al., 2009; Raz \& Gabis, 2009; Richardson, 2006; Scorza, et al., 2008; Yuen \& Sander, 2004), and others showing mixed (Amminger et al., 2007; Voigt et al., 2001; Yuen \& Sander, 2004) or neutral results (Chalon, 2009; Yuen, et al., 2005).

Although both omega-3s and omega-6s are of importance for normal body functioning, a large percentage of studies examining the effects of supplementation involve only omega-3s or a mixture of the two. Three possible explanations for this preference might include, 1) EFA deficiencies exhibited by individuals diagnosed with ADHD/ASD are largely omega-3 based (Stevens et al., 1995), therefore, the first attempt at treatment would be through supplementation of only omega-3s; 2) Omega-6s usually promote an inflammatory reaction and thus, omega-6 supplementation carries a risk for negative consequences, whereas omega-3 FAs are antiinflammatory and doses of greater than $20 \mathrm{mg} / \mathrm{kg}$ are well-tolerated within the body without negative side effects (DeGiorgio, Miller, Meymandi, \& Gornbein, 2008); and 3) Omega-3 may be the chosen supplement based on convenience. Typical EFA supplements consist of only an omega-3 blend (EPA/DHA) obtained from fish oil, which is inexpensive and easily obtainable. Some supplements consist of an omega-3/omega-6 mixture, however, the percentage of omega$3 \mathrm{~s}$ is consistently higher than the percentage of omega-6s. Both types of EFAs are vital components of the cell membrane, which affect membrane fluidity due to their chemical 
structure. Changes in membrane fluidity due to EFA supplementation are linked to numerous secondary areas of neuronal functioning, including neurotransmission, enzyme and ion channel regulation, and gene expression (Young, et al., 2000).

Studies have proven supplementation to be quite successful against ADHD symptoms (Antalis et al., 2006; Johnson, et al., 2009; Raz \& Gabis, 2009). A meta-analysis involving omega-3 supplementation in individuals suffering from ADHD found a significant increase in plasma omega-3 levels and a reduction in ADHD associated behaviours in individuals that received supplementation (Raz \& Gabis, 2009). Furthermore, research involving DHA supplementation alone revealed similar results with $26 \%$ of participants reporting a reduction in ADHD symptoms after three months of supplementation, while $47 \%$ of participants showed this improvement after six months of supplementation (Johnson, et al., 2009).

In contrast, a second meta-analysis investigating ADHD and omega-3 supplementation found that while individuals diagnosed with ADHD reliably showed reduced blood levels of DHA prior to treatment, supplementation did not increase these levels or lead to a reduction in ADHD type behaviour (Chalon, 2009). Voigt et al. (2001) also found that although supplementation of DHA over a four month period led to an increase in blood plasma omega-3 levels, there was no effect on the behavioural symptoms associated with ADHD. However, these discrepancies may have been due to the many variations in experimental design between these large patient-based studies, including differences in experimental design, mode of administration, duration of supplementation, and outcome measures. Thus, based on these results, the treatment of $\mathrm{ADHD}$ via omega-3 supplementation certainly warrants further investigation. 
The investigation into the efficacy of omega-3 FA supplementation as a treatment for epilepsy has also yielded mixed results (Cysneiros, et al., 2009; Ferrari, et al., 2008; Scorza, et al., 2008; Yuen \& Sander, 2004; Yuen, et al., 2005). Supplementation of a DHA/EPA mixture leads to an increase in blood concentrations of DHA and EPA, as well as reduced seizure susceptibility, however, decreased seizure frequency was only sustained for the first half of a twelve week study by Yuen et al. (2005). Long term studies have indicated more positive results. A thirteen month study involving DHA/EPA supplementation found increased blood serum levels of DHA accompanied by decreased seizure duration and severity in epileptic patients (Ferrari, et al., 2008; Scorza, et al., 2008; Yuen \& Sander, 2004). Furthermore, supplementation research has indicated associated treatment effects are due to increased neuroprotection associated with inflated omega-3 FA levels (Cysneiros, et al., 2009).

\section{Maternal Omega-3 Fatty Acid Supplementation}

Maternal health/diet is of utmost importance for fetal development. This is of particular necessity with respect to FAs intake during gestation, as the fetus must rely solely on maternal dietary intake at this time to receive appropriate levels of EFAs for proper neurodevelopment. Several studies have highlighted this relationship and results indicate dietary omega-3 intake during pregnancy has a direct influence on fetal neurodevelopment (Agale, et al., 2010; Donahue et al., 2009; Harris, Connor, \& Lindsey, 1984; Innis, 2007; Makrides et al., 2009), which is permanent (Church, Jen, Jackson, Adams, \& Hotra, 2009; Decsi \& Koletzko, 2005; Hanebutt, Demmelmair, Schiessl, Larque, \& Koletzko, 2008; Jen et al., 2009; Saste et al., 1998; van Goor et al., 2009). 
Prenatally, the fetus has access to maternally ingested or synthesized EFAs via placental transfer. Indeed medium positive correlations in omega-3 levels have been reported between maternal diet and maternal blood (.38), maternal diet and umbilical cord blood (.34) and maternal blood and umbilical cord blood (.37) (Donahue et al., 2009). This clearly suggests that not only does the fetus have access to these maternal EFAs but a significant maternal-offspring transfer does occur. Also, research investigating proper omega-3 nutrition during gestation has indicated early neurodevelopmental success, as measured by reduced latency to reach developmental milestones, is positively correlated with umbilical cord DHA levels (Bouwstra et al., 2006; Makrides et al., 2009).

Postnatally, maternal-infant EFA transfer occurs via lactation. Although throughout pregnancy nutrient levels available to the fetus are primarily associated with maternal dietary intake, postnatal infant EFAs are primarily obtained due to the breakdown of maternal fat stores (70\%) (Sauerwald et al., 2001). EFAs available for maternal-offspring transfer throughout breastfeeding, however, appear to be malleable to outside influences. Specifically, research has indicated omega-3 supplementation in lactating women significantly increased breast milk levels of omega-3s compared to non-supplemented individuals (Dunstan et al., 2007; Dunstan et al., 2003). Neo-natal research has investigated the relationship between maternal omega-3 breast milk levels and offspring plasma omega-3 levels both short and long term (Decsi \& Koletzko, 2005; Singh, 2005). Interestingly, as measured at three days and six months of age, offspring DHA levels showed a medium positive correlation with maternal DHA levels in the breast milk. Longitudinal maternal omega-3 supplementation studies found that at the age of four child mental processing scores showed a significant positive relationship with maternal DHA intake during pregnancy and breastfeeding. As well, these children showed greater psychomotor 
development and hand-eye coordination when compared to controls (Decsi \& Koletzko, 2005; Singh, 2005). These results indicate the ability of maternal omega-3 supplementation to affect early neurodevelopment in offspring leading to biological and cognitive changes in the developing fetus and young infant.

\section{$\underline{\text { Seizure-prone versus Seizure-resistant Rat Strains }}$}

The ability of the brain to undergo relatively permanent changes in structure/function in a short period of time following exposure to an outside stimulus directly demonstrates the brain's neuroplastic properties (Goddard \& Douglas, 1975). Although this concept is nearly dogmatic today, Goddard (1967; Goddard, McIntyre \& Leech, 1969) was one of the first to report this phenomenon in the late 1960 s based on observations pertaining to his learning, memory and kindling research. Specifically, kindling involves an initial stimulation of a brain structure with high frequency pulses of electrical current producing a sub-convulsive afterdischarge. Repeated exposure to that same stimulation intensity, however, produces progressively longer afterdischarges (AD), leading to the recruitment of secondary brain structures, such as the motor system. When this occurs, overt seizure behaviours are generated. The progressive nature of kindling is observed through the use of an electroencephalograph (EEG), and the associated observable behavioural manifestations, which have been classified into 5 progressive stages of seizure (Racine, Steingart, \& McIntyre, 1999). Stage 1 is defined as freezing behaviour with staring and mastication; stage 2, manifests with severe jaw mastication and drooling; stage 3, reflects mild to severe bilateral fore- and hind limb clonus without rearing; stage 4, appears as rearing with back extended, in addition to severe forelimb clonus; and, finally, stage 5, rearing with falling, is associated with a clonic/tonic convulsive seizure. Once animals have reached the 
"kindled" state (exhibited a stage 5 seizure), the resulting changes in brain structure/function and excitability are relatively permanent.

Animal models have often been used in research as a practical method of investigating human disorders. Over a decade ago, Racine et al. (1999) developed two rat strains with differential genetic predisposition to seizure sensitivity based on kindling rate, which is defined as the number of stimulations required to produce a stage 5 seizure. These strains were developed through selective breeding processes based on amygdala kindling rate; this selection ultimately produced seizure-prone fast kindling (FAST) and seizure-resistant slow kindling (SLOW) rats. The original parental population consisted of 24 Long Evans Hooded (LEH) $($ female $=12$, male $=12)$ and 24 Wistar rats $($ female $=12$, male $=12)$. Both strains were first kindled to one stage- 5 seizure and then bred appropriately based on their kindling rates; those with higher and lower kindling rates were mated within their own kindling rate groups. Pairings in the parental generation (P1) consisted of random LEH and Wistar crosses. All viable offspring resulting from these pairings in subsequent iterations (F1-F11) underwent identical kindling and mating procedures as occurred in P1, with the proviso that only rats more distantly related than second cousins were paired. Statistically significant differential kindling rates between the two developing strains were established by the sixth generation of pairings, yet the strains continued to be bred based on kindling rates until the eleventh generation. Since that time, the strains have not been re-derived, and continue to show highly reliable differences in kindling rates regardless of the brain structure being stimulated (Racine, et al., 1999).

Besides differential kindling rates, numerous behavioural differences have been reported between the seizure-prone and seizure-resistant strains, including differences in overall activity 
levels, impulsivity, learning and memory, attention, anxiety and aggression. Specifically, both in the home cage and novel environments, such as the Open Field (OF) paradigm, the seizure-prone FAST phenotype exhibit relative hyperactive behaviour (Gilby, Crino, et al., 2007; Gilby, Jans, \& McIntyre, 2009; Gilby, Thorne, Patey, \& McIntyre, 2007). On average, FAST rats are up to four times more active than their SLOW counterparts in the OF. Overnight examination of home cage activity levels using an activity monitor also revealed FAST rats move significantly more than SLOW rats when in their natural environment (Gilby, et al., 2009).

Differential hyperactivity/impulsivity-like behaviours have also been observed between the strains in the Restraint/impulsivity paradigm. As defined by the Merriam-Webster Online Dictionary (2010), impulsivity means to be "prone to act on impulse" with impulse being defined as "1) the act of driving forward with sudden force, 2) a force so communicated as to produce motion suddenly, 3) a propensity or natural tendency usually other than rational". When animal mobility is restricted through restraint, FAST rats struggle up to $40 \%$ of the time restrained, whereas SLOW rats struggle only $10 \%$ of that time (McIntyre et al., 1999). FAST rats appear to be unable to lie still and instead show repeated, sudden bouts of violent struggling, which is believed to represent an impulsive act in addition to hyperactive behaviour. This behaviour exhibited by the FAST strain is particularly relevant as both the SLOW and "normal" outbred rats (Long-Evan Hooded, Wistar) show some struggling at the beginning of the testing period but stop struggling completely in the latter half of the assessment (Gilby, Thorne, et al., 2007; Mohapel \& McIntyre, 1998). Arguably, the specific behaviour of the FAST rats can be described as "the act of driving forward with sudden force" or "a force so communicated as to produce motion suddenly". 
A second indication of enhanced impulsivity in FAST rats would be via examination of natural reproductive behaviour exhibited by the two strains. When introduced to a novel female in estrous, SLOW rats take an average of 40 seconds to first explore and then mount the female, whereas the FAST rats will attempt to mount within an average of 10 seconds. Indeed, strain differences are magnified when the rats were introduced to a novel female not in estrous. In that situation, SLOW rats explore but do not attempt to mount the female (normal behaviour based on rat social cues), whereas FAST rats, on average, still attempt to mount the female within 10 seconds (Gilby, Crino, et al., 2007). Thus, FAST rat behaviour indicates social cues are not highly relevant to FAST rats, likely due to a lack of attention and/or the inability to recognize cues that normally mediate social behaviours - a relationship often paralleled in individuals with ASD (Richardson, 2006; Wing \& Potter, 2002).

In addition to FAST rats not following appropriate behavioural cues in a sexual paradigm, further evidence of abnormal social behaviour has been observed in FAST pups. Reinhart et al. (2004) reported that FAST pups maintain infantile play behaviours much later in development than SLOW pups. FAST rats also often exhibit repetitive behaviours such as circling behaviours in their home cage as well as excessive grooming of themselves and/or their pups (Gilby, Crino, et al., 2007).

Strain differences have also been observed with respect to learning and memory (Anisman \& McIntyre, 2002; Azarbar, McIntyre, \& Gilby, 2010; Gilby, Crino, et al., 2007; Gilby, et al., 2009; Gilby, Thorne, et al., 2007; McIntyre \& Gilby, 2007; McIntyre, Kelly, \& Dufresne, 1999; McIntyre, Poulter, \& Gilby, 2002). Spatial and place learning capacity as well as both short and long term memory have been assessed in the strains using the Morris Water 
Maze (MWM). Although these strains do not tend to differ in latency to locate the platform on the first testing day, SLOW rats show a higher rate of learning and greater memory retention compared to the FAST rats across the subsequent testing period, as indicated by reduced latency to reach the platform assessed over numerous testing trials. The FAST strain also show a further increase in latency to reach the platform when distracting cues are introduced (hanging cues), indicating FAST rats may have reduced attention compared to SLOW rats (Anisman \& McIntyre, 2002).

Differential anxiety levels and tendency towards aggressive-like behaviours have also been associated with the two strains. SLOW rats spend a greater amount of time in the closed arms of the Elevated Plus Maze compared to FAST rats, indicating a greater level of anxiety. While the FAST rats typically exhibit lower levels of anxiety, they show a higher incidence of aggressive type behaviours, as illustrated by a higher level of fighting behaviours with same-sex sibling in their home cage compared to the SLOW rats (Gilby \& McIntyre, 2007).

Thus the collection of behaviours exhibited by seizure-prone (FAST) rats, include an increase in activity and aggression levels as well as impulsive and repetitive behaviours, along with a decrease in learning, memory, anxiety and attention compared to seizure-resistant (SLOW) rats. In addition, FAST rats also ignore or lack the ability to pick up on normal social cues and exhibit abnormal social interactions compared to SLOW rats. Remarkably, these behaviours are highly reminiscent of ADHD/ASD type symptomology, thus indicating a possible link between the manifestation of seizure disorders and ADHD/ASD within the FAST strain. Neurodevelopmental and neuroanatomical research regarding the strains revealed further indication that FAST rats show a high comorbidity with ADHD/ASD type symptomology. With 
regards to neurodevelopment, SLOW pups open their eyes and show cliff avoidance plus righting reflex behaviours significantly earlier than FAST pups (St. Onge, unpublished data), a finding that is paralleled in clinical research where children with ADHD/ASD/SD take longer to reach developmental milestones than healthy individuals (Hanebutt, et al., 2008; Innis, 2007; Makrides, et al., 2009). Also indicative of improper neurodevelopment, FAST rats reliably show a decreased in white matter volume compared to SLOW rats, a neuroanatomical difference that appears to reflect myelination differences between the strains (Gilby, Crino, et al., 2007; Gilby, Thorne, et al., 2007).

Lastly, recent experimentation has revealed that FAST rats have lower circulating levels of specific EFAs compared to SLOW rats (Gilby, unpublished data). Although strain differences include a reduction of complex omega-3 FAs DHA (22:6(n-3)) and EPA (22:5(n-3)) compared to the SLOW rats, suggesting the strains show metabolic differences in lipid handling. Although the underlying lipid handling mechanism is unclear, individuals diagnosed with ADHD/ASD also show decreases in omega-3 FAs compared to healthy controls which, in association with behavioural, neuroanatomical and neurodevelopmental evidence, suggests the seizure-prone FAST rats may be a possible model for investigating the relationship between omega-3 FA and the manifestation of ADHD/ASD and seizure disorders.

Recent experimentation (Gilby, et al., 2009) investigated the effect of omega-3 supplementation in adult rats. Although omega-3 supplementation did not significantly alter hyperactivity, learning deficits or the normal heighten seizure susceptibility in FAST rats, it significantly lowered their levels of measured impulsivity to levels normally observed in the SLOW rats (Gilby, et al., 2009). Supplementation of omega-3s in SLOW rats did not result in 
differential behavioural patterns from SLOW controls, however, it did increase their relative seizure susceptibility and reduced DHA/EPA FA levels to those associated with the FAST strain (Gilby, et al., 2009). Based on these results, indicating that omega-3 supplementation in the adult rat leads to measurable changes in behaviour and seizure susceptibility, the next step would be to examine if maternal omega-3 supplementation would prevent ADHD/ASD type behaviour and reduce seizure susceptibility in offspring.

\section{Purpose of Study}

There is clear evidence of essential FA irregularities in ADHD/ASD and epilepsy, which has led to omega-3 FA supplementation being employed as a treatment option for these disorders. However, there have been no studies to date that use maternal supplementation as a method for prevention of these disorders. Given the relationship between maternal and fetal plasma omega-3 FA levels, the purpose of this thesis is to determine whether maternal supplementation during both pregnancy and lactation will improve ADHD/ASD-like characteristics in FAST rats. Thus, FAST and SLOW mothers received high doses of omega-3 EFAs via oral (gavage) fish oil supplementation or treatment (FTx, STx) throughout pregnancy and lactation. Control mothers of both strains received either gavage to control for the procedural stressor (FC-G, SC-G) or no gavage (FC, SC). Adult offspring from these mothers underwent behavioural and kindling testing in order to determine if omega-3 supplementation during gestation and infancy affects the traits that normally characterize these strains as adults. It is hypothesized that maternal fish oil (omega-3) supplementation during gestation and throughout lactation will result in permanent neurological alterations in the FAST strain, and by extension, will reduce ADHD/ASD-like behaviours and seizure sensitivity. Behavioural and 
kindling results in line with those predicted would indicate potential for maternal omega-3 supplementation in correcting aberrant developmental trajectories, preventing the manifestation of FAST traits altogether. The effect of maternal omega-3 supplementation in the non-deficient SLOW rats is not easily predicted, however, based on reports indicating that omega-3s are tolerated well by the body even at very high levels (Gochfeld \& Burger, 2005), negative effects are not expected.

\section{Experimental Design}

A 2 (Strain) x 3 (Group) experimental design was implemented for this study. FAST and SLOW rats were randomly assigned to three groups per strain - two controls and one treatment. Mothers in the treatment group received daily omega-3 supplementation beginning two weeks prior to gestation, which continued until the pups were weaned on post natal day (PND) 23 . Control mothers were divided into two groups - one group received a sham gavage procedure while the second group remained untreated. Once pups were weaned, they were double housed and allowed to mature until PND 60 when behavioural testing commenced. Testing included 4 consecutive days of Open Field (OF), followed by 5 consecutive days of Morris Water Maze (MWM), and concluded with one day of restraint testing. After behavioural testing was completed, offspring underwent amygdala electrode implantation surgery and later kindling until 6 stage- 5 seizures were recorded. Subsequent to the kindling procedure, animals were perfused, and their brains were removed and sliced in order to determine electrode placement accuracy. The ideal number of offspring would be 10 per group/per strain (from 60 mothers), with a maximum of two male pups per litter being tested. Offspring numbers were expected to be dependent on pup viability as well as possible effects of gavage and treatment stress. 


\section{Material and Methods}

\section{$\underline{\text { Animals }}$}

A total of 80 female rats and 90 male rats were used in this study. Forty male rats were used for breeding purposes (20 SLOW Adults, 20 FAST Adults), while 50 offspring pups (29 SLOW pups, 21 FAST pups) were used in this study. All animals were bred and raised in the Carleton University Life Sciences Facility and treated in accordance with guidelines from the Canadian Council on Animal Care (CCAC) and a research protocol approved by the Carleton University Animal Care Committee. All rats were housed in opaque shoebox cages (44 cm long x $24 \mathrm{~cm}$ wide x $20 \mathrm{~cm}$ deep) and given ad libitum access to food (5075, Charles River, StConstant, Quebec, Canada) and water. Housing rooms were maintained on a 12:12 light/dark cycle (lights came on at 8 a.m.), at 21 degrees Celsius, and between $40-60 \%$ humidity for the duration of the experiment. All possible efforts were made to minimize animal usage and their discomfort.

\section{$\underline{\text { Treatment }}$}

FAST and SLOW female rats were randomly assigned to control and treatment conditions (33 FAST TX, 17 FAST C-G, 15 SLOW TX, 15 SLOW C-G). In order to achieve the highest likelihood of determining if omega-3 FA supplementation would affect neurodevelopment in this experiment, pups were exposed to omega-3 supplementation for the equivalent of approximately 2.5 human years ( 6 weeks) at a high dose $(20 \mathrm{mg} / \mathrm{kg})$ (Bioriginal, Fish Oil Blend: 18/12 EPA/DHA, Saskatoon, Canada). Maternal rats were supplemented beginning two weeks prior to mating until pups were weaned at postnatal day (PND) 23. Fish oil was administered orally via gavage using disposable plastic gavage tubes $(13 \mathrm{G} \times 90 \mathrm{~mm}$, 
Insotech Solomon). Treatment was given by the use of gavage in order to administer a specific dose, avoid oxidation of the fish oil and parallel the human supplementation process (typically ingested in pill form). In order to control for the possible confounding stress associated with the gavage procedure, one group of female control rats was sham gavaged daily in a manner identical to the treated rats, while a second group of control females were left unhandled during the treatment period (these SC and FC mothers not included in animal numbers). Offspring were housed with their mother until weaning, after which pups were allowed to mature before undergoing a series of behavioural tests previously shown to be reliable in presenting strain differences. Following the triad of behavioural tests, rats underwent amygdala implantation surgery and were kindled in order to determine if maternal omega-3 supplementation led to kindling or other seizure characteristics that were different between seizure-prone and seizureresistant strains.

Due to fertility issues in some groups, total offspring numbers $(\mathrm{N}=50)$ represent the culmination of results obtained from testing four cohorts. Cohorts 1 and 4 had at least one litter representing each of the six treatment groups, however, pairings in cohorts 2 and 3 did not result in any viable litters of FAST treated offspring.

\section{$\underline{\text { Behavioural Tests }}$}

One or two male pups (PND 73-90) from each litter were behaviourally assessed using our standard test battery, which reliably produces differential behaviour patterns in FAST and SLOW rats. Specifically, each rat completed 4 consecutive days of Open Field (OF) testing followed by 5 days of the Morris Water Maze (MWM) and one day of Restraint (R) testing. All animals underwent the behavioural testing in the same sequence. 


\section{Open Field}

The open field testing apparatus comprised of a white open-top box with $50 \mathrm{~cm}$ wooden walls and a $100 \times 100 \mathrm{~cm}$ plexiglass floor. The floor was gridded into 25 equal $20 \times 20 \mathrm{~cm}$ squares with black tape. A video camera was mounted directly above the testing apparatus so behaviours could be recorded and re-scored at a later date if necessary. On each testing day, rats were placed in the center square and the latency to move from the center square, as well as the number of lines crossed within the 10 minute period were recorded (Gilby, Thorne, et al., 2007). The amount of urine/feces excreted was also recorded as an index of relative stress levels in the novel environment. The apparatus was cleaned with 50\% ethanol and allowed to dry between test subjects.

\section{Morris Water Maze}

Each rat was tested in the MWM 24 hours after completion of the Open Field. The water maze consisted of a white polypropylene pool with a depth of $80 \mathrm{~cm}$ and a diameter of $158 \mathrm{~cm}$. The pool was filled to $55 \mathrm{~cm}$ with a water temperature of 21-23 degrees Celsius and made opaque by the addition of white powder paint. The pool was divided into four equal quadrants and a clear circular Plexiglass platform (14 cm diameter-) was placed in the middle of the second quadrant and submerged $2 \mathrm{~cm}$ below the waterline for the first four days of testing. On the fifth and final day of testing (swim test), the platform remained in the same location, however, was raised $2 \mathrm{~cm}$ above the waterline, covered in a dark towel for traction or gripping by the rat, and the latency to reach and mount the visible platform was recorded.

Each day of testing involved four trials and each trial began by placing the rat in the pool, facing the wall, in one of the four quadrants (i.e., north, south, east or west). Latencies to locate 
and sit atop the submerged platform were recorded. Starting positions were randomly selected with the provision that all starting positions were used each day, and the same quadrant order was used for all animals on a given day. Rats that did not locate the platform after sixty seconds were manually placed on the platform. Each trial was followed by a 60 second interval wherein the rats were placed in a warm, dry, clear shoebox cage. Trials were videotaped from overhead to permit re-scoring if necessary.

\section{$\underline{\text { Restraint }}$}

All rats were assessed in the Restraint test the day following completion of the MWM. Each rat was placed in the wide end of a triangular plastic restraint bag with a small hole in the narrow end to allow the rat's nose to protrude. Once securely in the bag, the wider end was taped shut allowing the rat's tail to protrude. The restraint bag was made snug enough to allow for only minimal movement of the rats. Each 'bagged' rat was then placed on a table for 10 minutes of observation. Time spent struggling during the first five minutes as well as the total time spent struggling was recorded. The number of vocalizations and amount of urine/feces excreted was also recorded. Rats were tested one at a time and returned to their home cage immediately following removal from the restraint bag.

\section{Kindling Surgery}

Rats were deeply anesthetized using an intraperitoneal injection (i.p.) of sodium pentobarbital $(60 \mathrm{mg} / \mathrm{kg})$ and supplemented with isoflorane if needed. A ground and 5 jeweller's screws were inserted into the skull prior to electrode implantation to assist in the head cap assembly (Molino and McIntyre, 1972). The ground consisted of a male Amphenol pin previously soldered to a two inch wire attached to a jeweller's screw. 
Each bipolar stimulating/recording electrode ( 2 twisted strands of $0.127 \mathrm{~mm}$ diameter Diamel-insulated Nichrome wire attached to two male Amphenol pins) were stereotaxically implanted in the amygdala. The stereotaxic co-ordinates (Paxinos and Watson, 1986) used for amygdala implantation were $2 \mathrm{~mm}$ posterior to bregma, $5 \mathrm{~mm}$ lateral to the midline and $8.5 \mathrm{~mm}$ ventral to the surface of the skull. Once the electrodes were in place and secured with dental acrylic, the attached Amphenol pins were inserted into a plastic head cap and again secured with dental acrylic (Molino and McIntyre, 1972). Following surgery, rats receive a subcutaneous injection of meloxicam (Metacam, $0.2 \mathrm{mg} / \mathrm{kg}$ ) and were placed in a clean cage on a heating pad. Once active again, all rats were given mash and returned to the colony room for a two week recovery period prior to seizure afterdischarge threshold determination.

\section{Afterdischarge Threshold Determination \& Kindling Procedure}

Two weeks following surgery, the afterdischarge (AD) threshold (ADT) was determined in the right amygdala, and twenty four hours later, the ADT was determined on the contralateral side. The $\mathrm{ADT}$ is defined as the minimum stimulus intensity required to provoke an $\mathrm{AD}$, a spike-and-wave discharge that outlasts the stimulus by $2 \mathrm{~s}$ or more (McIntyre, et al., 1999). To determine the threshold, a $2 \mathrm{~s}, 60 \mathrm{~Hz}$ sine wave stimulus of progressively increasing intensity (25, $35,50,75,100,150,200,250,300,350 \mu \mathrm{A})$ was presented until an $\mathrm{AD}$ was triggered. The inter-stimulus interval was 1 min. Kindling began 24 hours following ADT determination and was achieved via daily unilateral stimulation of the selected amygdala (pre-determined based on its ADT, i.e., lowest intensity), at the appropriate stimulus intensity, until six stage -5 (Racine, et al., 1999) generalized convulsive seizures were elicited. Once kindling was complete, the ADT 
was re-determined in the kindled amygdala in the manner previously described. Twenty four hours later, the ADT was re-determined for the contralateral amygdala.

The ADT intensity and associated AD duration are measures of importance as they reflect local amygdala excitability. The number of daily stimulations required to elicit the first stage-5 seizure (kindling rate) was also assessed for each animal, as well as the cumulative AD duration required to elicit the first stage- 5 seizure. These latter parameters are relevant to the ease of network recruitment, and are well known to be different in the seizure-prone and seizureresistant rat strains. The behaviour of the rats during the kindling trials, including the severity of seizure, as indexed by the Racine scale (Racine, 1972), was recorded daily. Measures used to gauge generalized seizure severity (i.e., stage 5 seizures evoked in fully kindled rats) included the latency to forelimb clonus, duration of behavioural seizure, duration of stage 5 clonic seizure behaviour, and cumulative AD duration. These measures were recorded for each of the 6 stage-5 trials.

\section{$\underline{\text { Histology }}$}

At the end of the experiment, all rats were perfused intracardially with $0.9 \%$ saline followed by $4 \%$ paraformaldehyde. Brains were left in situ for twenty-four hours prior to extraction and stored in $4 \%$ paraformaldehyde. Brains were then transferred to $30 \%$ sucrose for at least 3 days prior to freezing and sectioning on a sliding microtome. Forty micron coronal sections were collected through the electrode tracts, then mounted on gelatine-coated slides and stained with cresyl violet to confirm electrode tip placement in the amygdala. 


\section{$\underline{\text { Cresyl Violet }}$}

Sections were dehydrated in a series of steps involving placement in increasing ethanol concentrations $(70,75,95,100 \%, 2$ minutes each), defatted with Clearene ( 3 minutes), rehydrated in decreasing ethanol concentrations and stained with $0.1 \%$ Cresyl Violet acetate (3 minutes). The sections were then rinsed twice in distilled water and placed in a differentiator for 2-3 minutes. The differentiator consisted of $0.8 \%$ glacial acetic acid. Sections were then dehydrated once more through a series of increasing ethanol concentrations. Finally, the sections were placed in Clearene, once again, until cover slipped. The sections were cover slipped using Permount and electrode placements were visualized using a Leica light microscope. Data from 6 subjects was removed from subsequent analysis as visual analysis indicated stimulating electrodes tip was not in the amygdala.

\section{$\underline{\text { Blood Plasma Extraction }}$}

Following 1 hour of food deprivation, blood was collected from all mothers on weaning day (PND 23). Blood was extracted from the heart via cardiac puncture and collected in $5 \mathrm{ml}$ lithium-heparin spray coated tubes (VWR International, LLC). Following centrifugation at 2800 rpm for 10 minutes at $4^{\circ} \mathrm{C}$, supernatant samples $(500 \mu \mathrm{l})$ were collected in an Eppendorf tube and stored at -20 degrees Celsius until shipped to the Animal Health Laboratory at the University of Guelph (Ontario, Canada) for free fatty acid analysis.

\section{Plasma Analysis}

In order to confirm global differences in fatty acid levels between strains, and determine if maternal omega-3 fatty acid supplementation resulted in differential fatty acid levels plasma 
concentrations, non-esterified fatty acids (NEFA) were compared between the six groups measured at the Animal Health Laboratory, University of Guelph (Ontario, Canada).

\section{$\underline{\text { Statistical Analysis }}$}

Open Field, MWM and Restraint performances were compared between groups using repeated measures ANOVA. With regards to kindling and blood plasma NEFA levels, all measures were compared between groups using ANOVA. Follow-up comparisons of simple effects for significant interactions were conducted using Bonferroni corrected t-tests. Significant values were set at $P<0.05$. 


\section{$\underline{\text { Behavioural Results }}$}

Open Field Test

The number of lines crossed within a given timeframe is used to index relative levels of locomotor activity in a novel environment (Boeck et al., 2009; Lalonde \& Strazielle, 2009; Tang, Wanchoo, Swann, \& Dafny, 2009). In this study, repeated measures ANOVA revealed a significant main effect of strain $[F(1,45)=65.696, p<.0001]$ where $F A S T$ rats crossed a greater number of lines over the 4 days than Slow rats. Within the FAST strain, both treatment and procedural effects were revealed on the second day of testing $[F(2,19)=4.97, p<0.05]$ where, although non-gavaged controls $(\mathrm{p}<0.05)$ and omega-3 supplemented $(\mathrm{p}<0.01)$ rats showed similar performance, both were significantly less active than gavaged control FAST rats.

Habituation also typically differs between FAST and SLOW rats (Gilby, et al., 2009) and involves a reduction in activity levels over trials as the animal habituates to the novel environment. Statistical analysis of this measure indicated a significant strain $\mathrm{x}$ day interaction $[\mathrm{F}(3,135)=6.418, \mathrm{p}<0.001]$, which reflects habituation by SLOW, but not FAST, rats over testing days. Once again, a non-significant effect of treatment $[\mathrm{F}(2,45)=0.814, \mathrm{p}=0.561$, n.s. $]$ indicated omega-3 supplementation did not affect habituation in either strain. See Figure 2. 


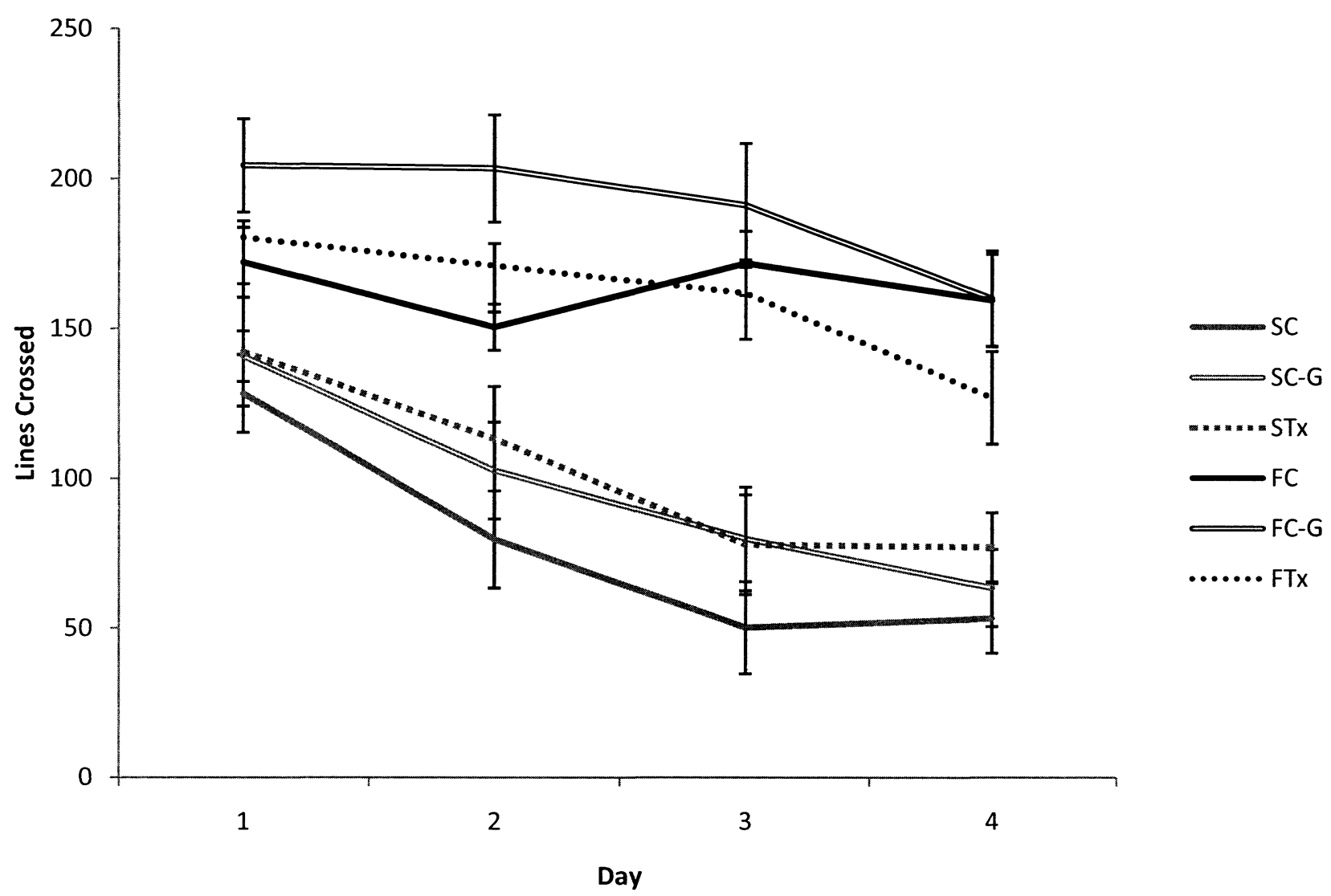

Figure 2: Relative activity levels in the open field paradigm. Mean (+/- S.E.M.) number of lines crossed by FAST versus SLOW control and treated rats over a 10 minute exposure to the open field paradigm across four days. Significant strain difference. $P<0.05$. 
Morris Water Maze (MWM)

The primary indication of relative learning and memory capacity in the MWM involves the latency to locate and mount a hidden (submerged) platform over successive trials. Typically, subsequent to the initial day of testing, SLOW rats show enhanced performance relative to FAST rats (Anisman \& McIntyre, 2002; Gilby, Crino, et al., 2007). In this study, a two way repeated measures ANOVA revealed a significant strain effect $[F(1,39)=32.153, p<0.0001]$, confirming the impoverished FAST performance levels, but no main effect of treatment when all 6 groups were included in the analysis. However, when analysis was confined within strains, maternal omega-3 fatty acid supplementation was found to significantly reduce latencies in FAST rats $[F(2,18)=60.984, p<0.0001]$. Indeed, on the $4^{\text {th }}$ day of testing omega- 3 supplemented FAST rats had a greater latency to reach the platform that non-gavaged controls $(\mathrm{p}<0.0001)$ who also took significantly more time to locate the platform than the gavaged-control rats (FTx. p $<0.0001$, FC $\mathrm{p}<0.002)$. See Figure 3.

An investigation of daily strain and treatment effects across trials revealed strains reached the platform in the same amount of time on the first day of testing $[F(1,39)=2.65, p=0.116$, n.s.], however, significant differences were revealed across all subsequent days (day $2[F(1,39)=9.609$, $\mathrm{p}<0.01]$, day $3[\mathrm{~F}(1,39)=21.82, \mathrm{p}<0.0001]$ and day $4[\mathrm{~F}(1,39)=45.673, \mathrm{p}<0.0001])$, where SLOW rats located the platform faster than FAST rats. Clearly, no effect of treatment was detected over the initial three days of testing (day $1[F(2,39)=.840, p=.439$, n.s. $]$, day $2[F(2,39)=0.214, p$ $=0.809$, n.s. $]$ and day $3[\mathrm{~F}(2,39)=0.044, \mathrm{p}=0.97$, n.s. $])$, or in SLOW rats on the fourth day of testing $[F(1,22)=3.748, p=0.0658$, n.s. $]$. On the fourth day of testing, however, omega-3 
supplementation significantly decreased latency to reach the platform in FAST rats compared to within-strain controls $[F(1,19)=37.622, \mathrm{p}<0.0001]$. See Figures 4-7.

On the fifth and final day of testing, the platform was raised above the waterline to enable visualization. This served as a 'swim test' to ensure similar swim speeds amongst groups when the memory components of the task were removed. Importantly, no strain $[F(1,39)=2.430$, $\mathrm{p}=0.127$, n.s. $]$ or treatment $[\mathrm{F}(2,39)=1.070 \mathrm{p}=0.353$, n.s. $]$ effects were evident on Day 5 , indicating similar swimming abilities across all groups and treatments. See Figure 8. 


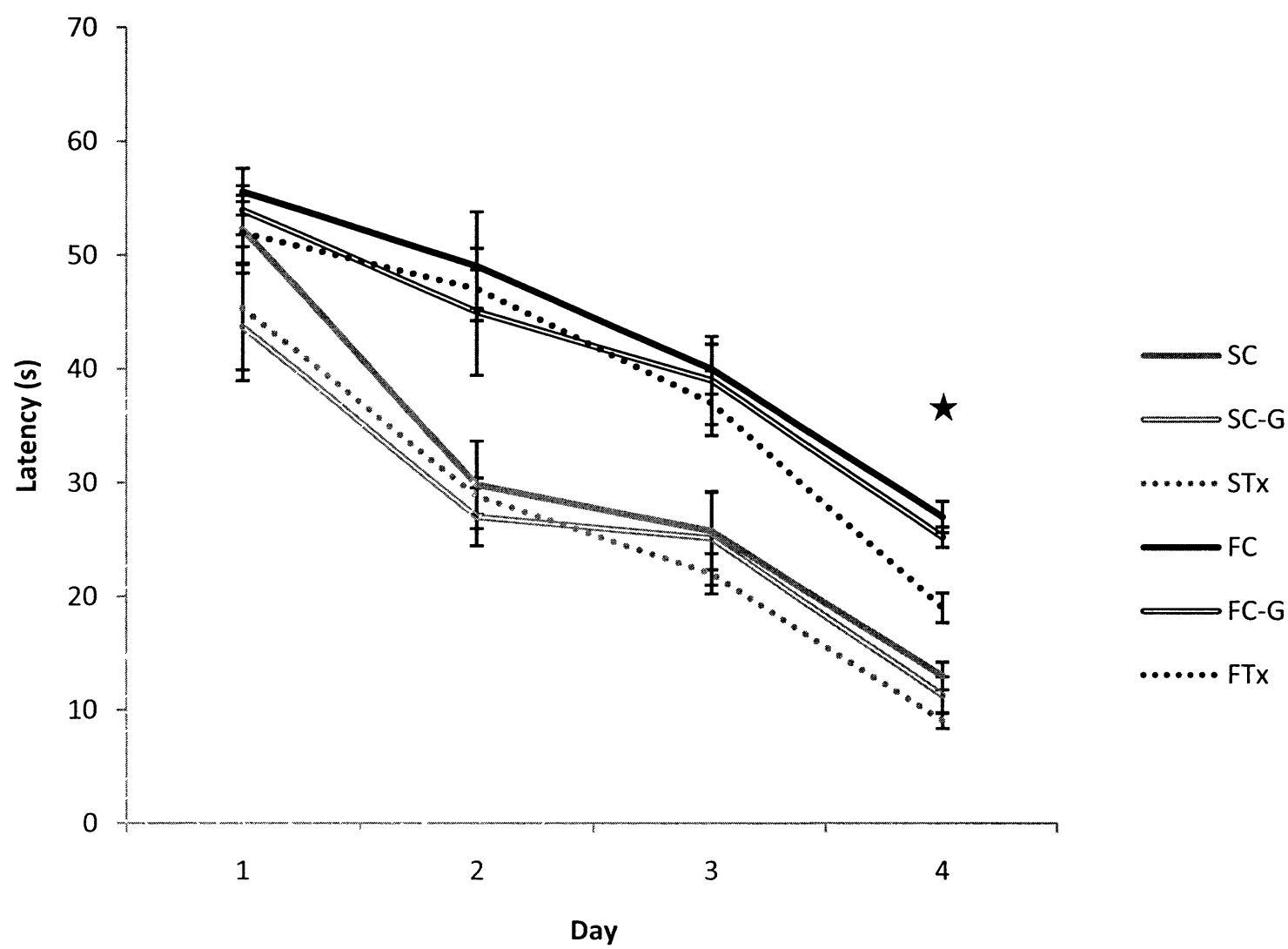

Figure 3: Relative learning and memory levels in the MWM. Mean (+/- S.E.M.) latencies to reach the platform for FAST versus SLOW control and treated rats across four testing days in the Morris Water Maze paradigm. Significant strain difference. $P<0.05$.

* Signficant difference between FAST treated and within strain controls. $P<0.05$. 


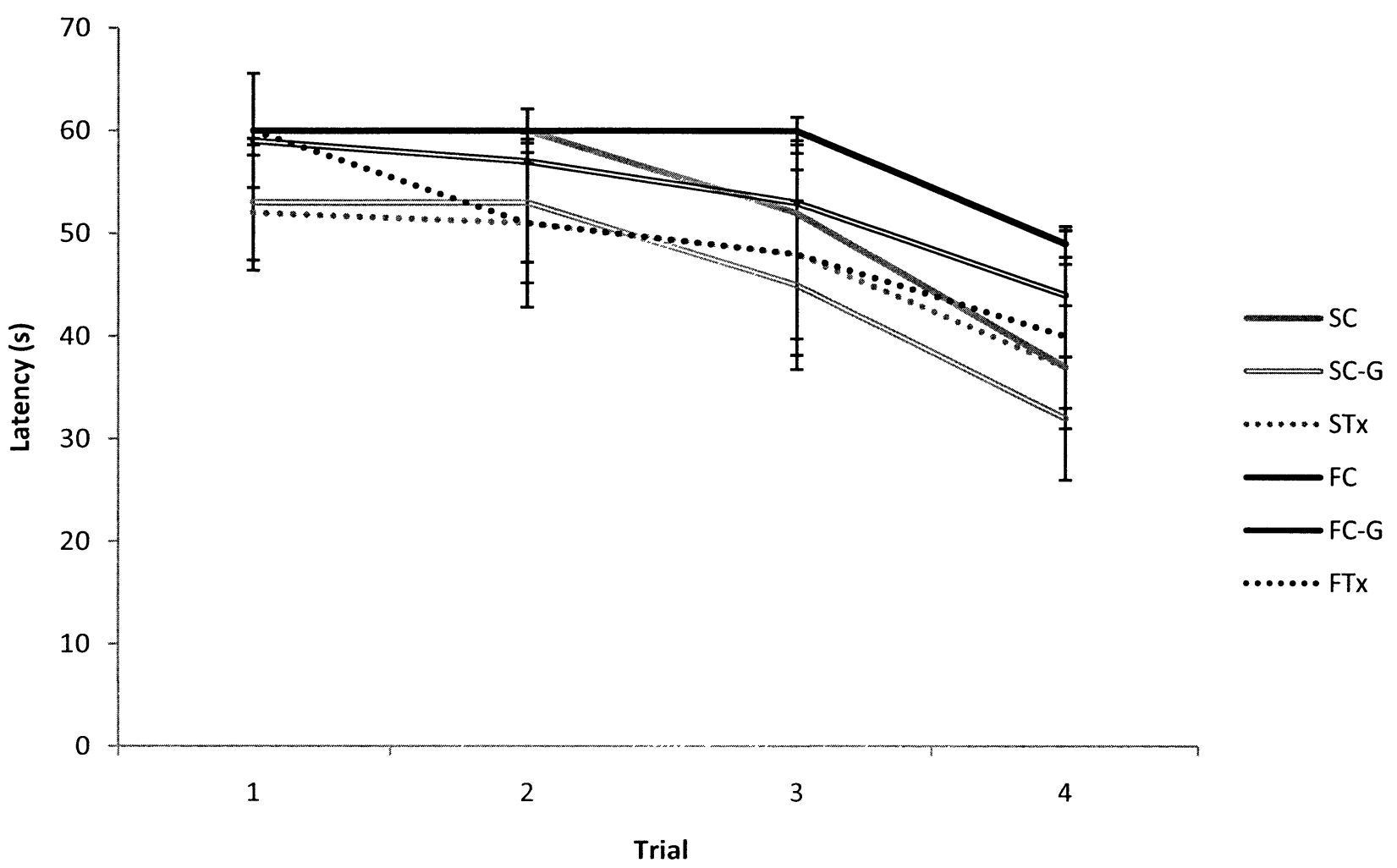

Figure 4: Relative learning and memory levels on Day 1 in the MWM. Mean (+/- S.E.M.) time required to reach the platform for FAST versus SLOW control and treated rats across four trials on the first testing day in the Morris Water Maze paradigm. 


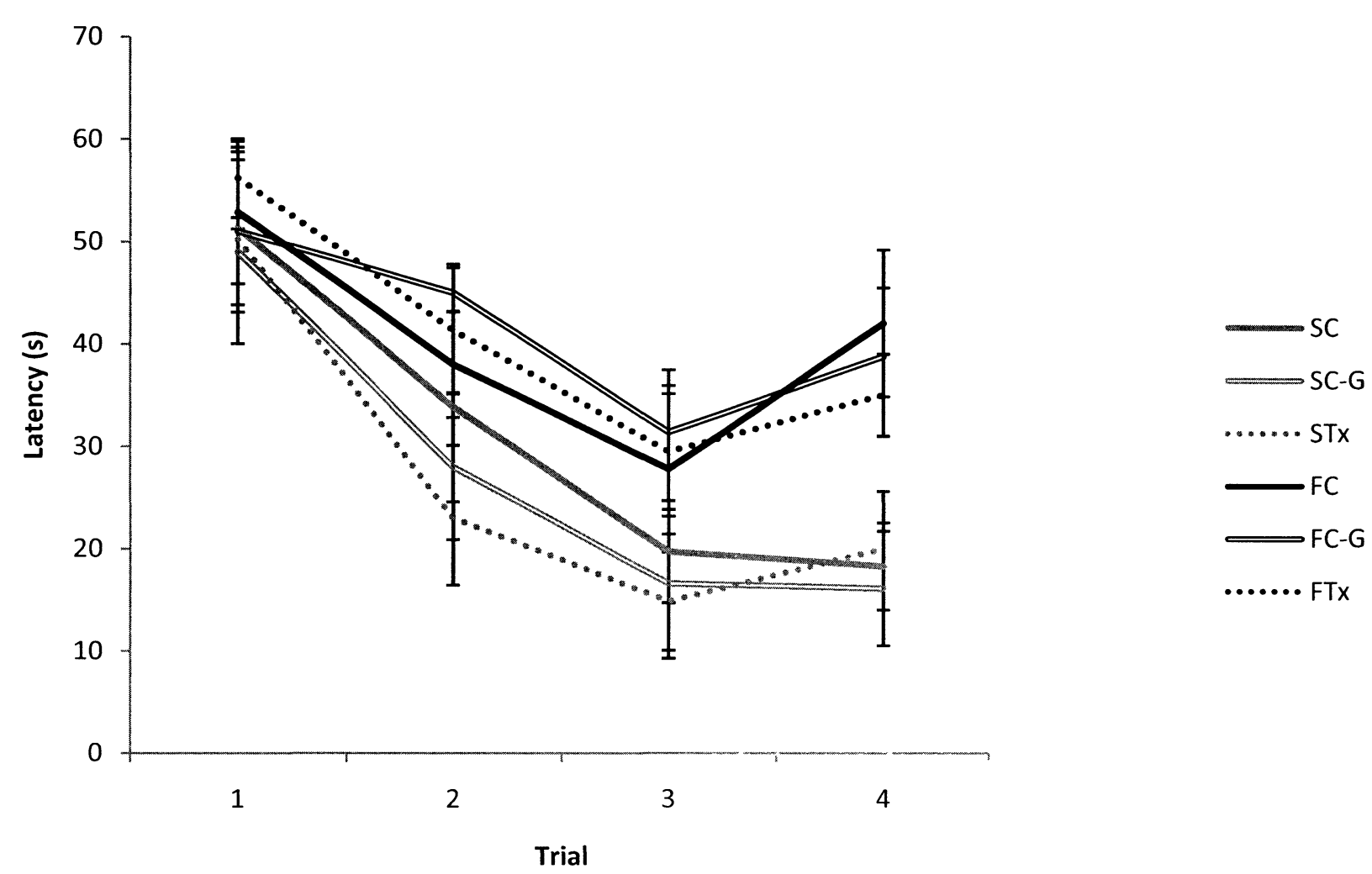

Figure 5: Relative learning and memory levels on Day 2 in the MWM. Mean (+/- S.E.M.) time required to reach the platform for FAST versus SLOW control and treated rats across four trials on the second testing day in the Morris Water Maze paradigm. Significant strain difference. $P<0.05$. 


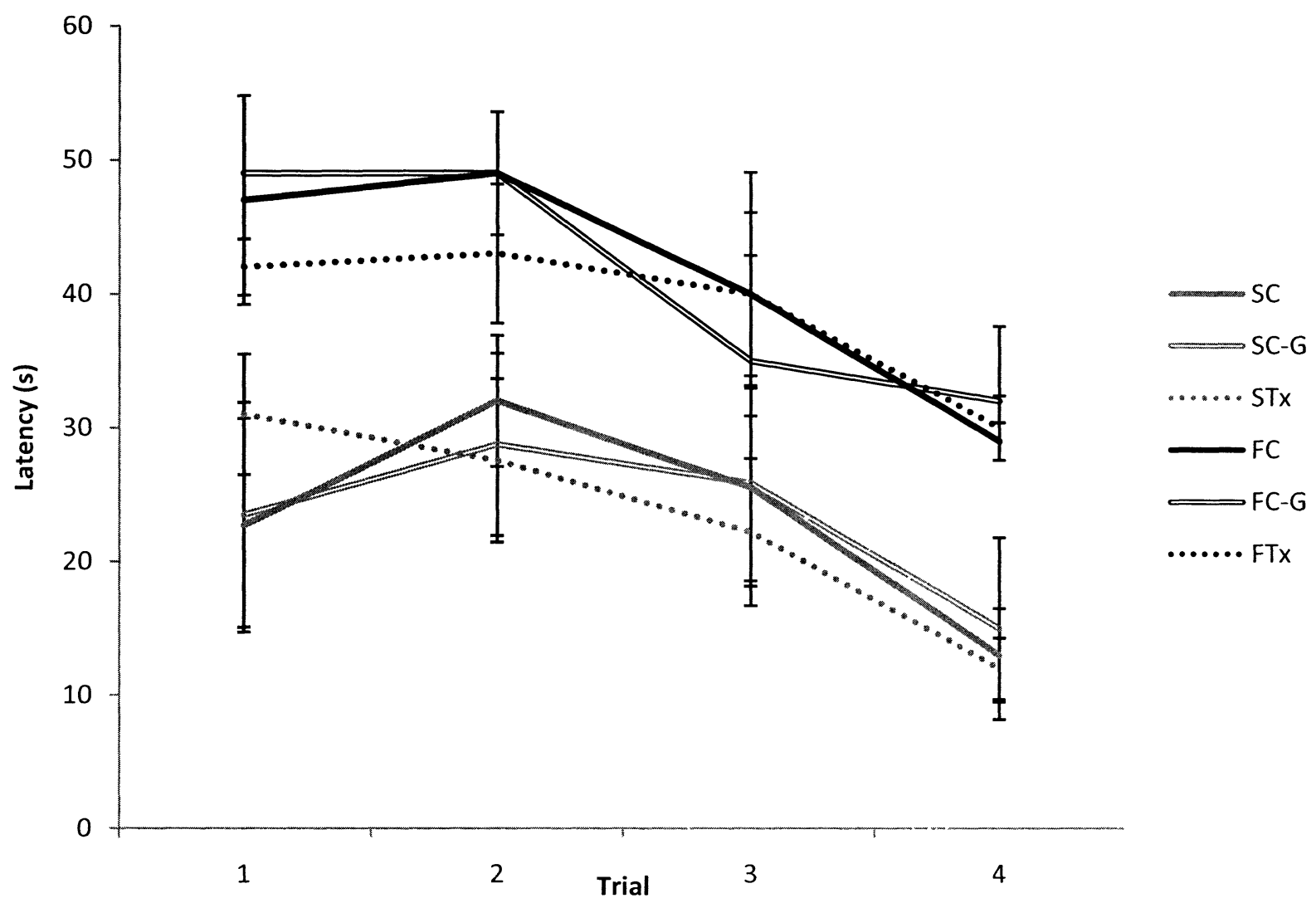

Figure 6: Relative learning and memory levels on Day 3 in the MWM. Mean (+/- S.E.M.) time required to reach the platform for FAST versus SLOW control and treated rats across four trials on the third testing day in the Morris Water Maze paradigm. Significant strain difference. $P<0.05$. 


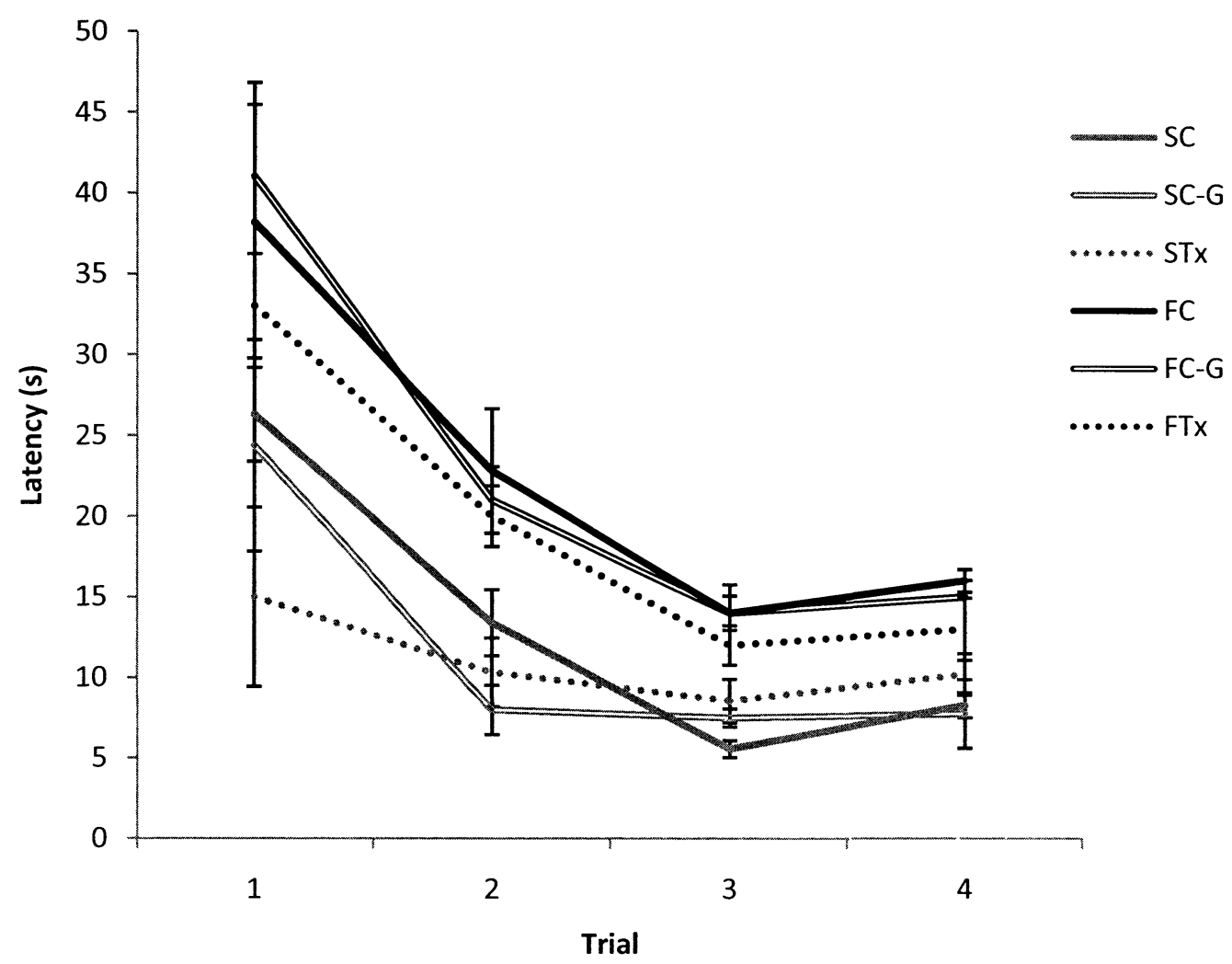

Figure 7: Relative learning and memory levels on Day 4 in the MWM. Mean (+/- S.E.M.) time required to reach the platform for FAST versus SLOW control and treated rats across four trials on the fourth testing day in the Morris Water Maze paradigm. Significant strain difference. $P<0.05$. 


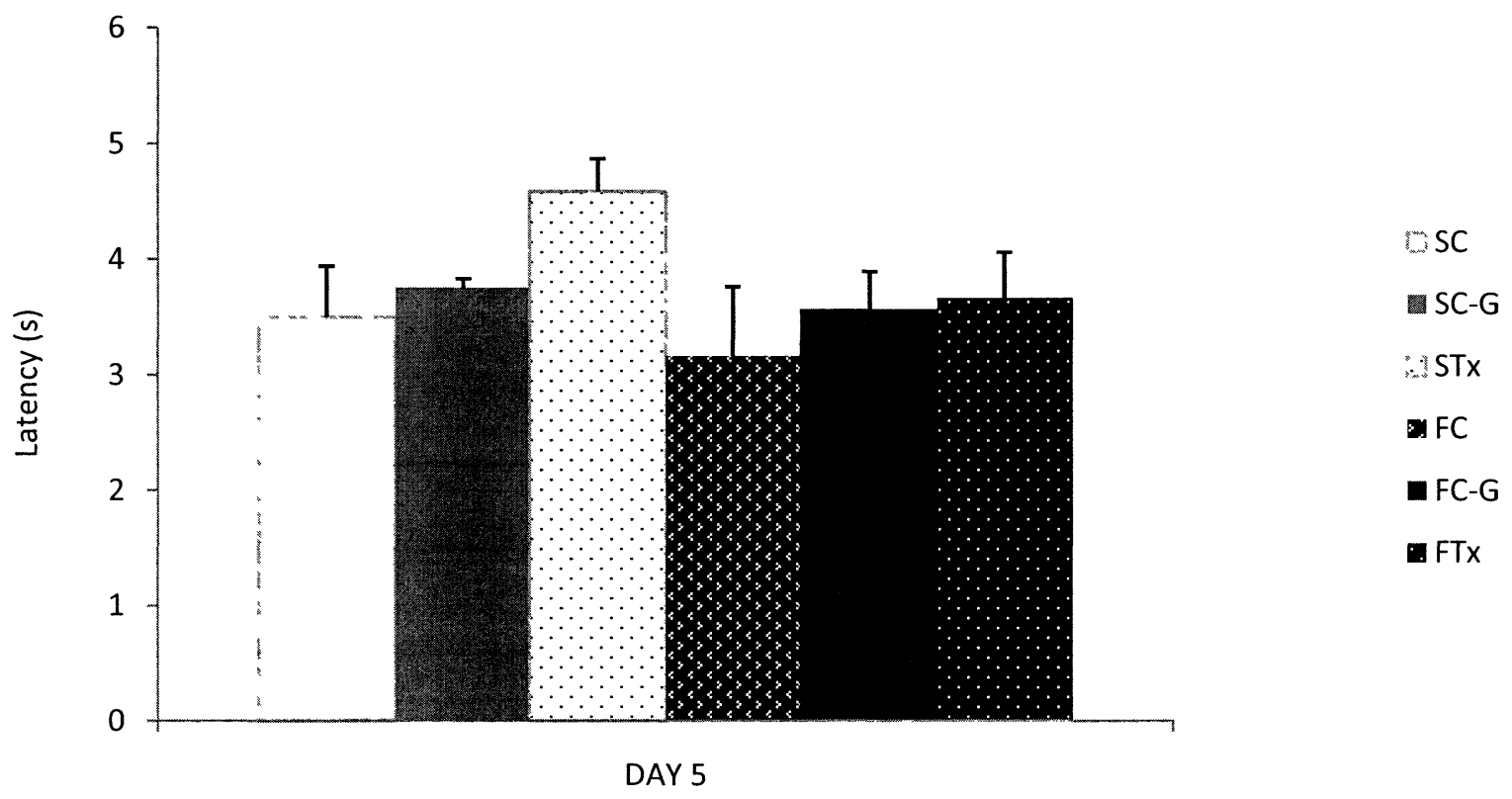

Figure 8: Relative swimming abilities in the MWM swim test. Mean (+/- S.E.M.) time required to reach the elevated platform for FAST versus SLOW control and treated rats on the fifth day of testing. 
Restraint

The tendency of FAST rats to struggle more than SLOW rats in the restraint paradigm has been consistently reported by previous studies (Anisman \& McIntyre, 2002; Gilby, Crino, et al., 2007; Gilby, et al., 2009) and is used to index relative impulsivity/hyperactivity. In this study, a two way ANOVA revealed strain $\mathrm{x}$ treatment interactions with regards to the percentage of total time rats spent actively struggling across the 5 minute test trial $[\mathrm{F}(2,39)=9.049, \mathrm{p}<0.001]$. Post hoc analysis confirmed SLOW rats struggled significantly less than FAST rats across the testing period. Furthermore, omega-3 supplementation led to a significant reduction in FAST struggling behaviour relative to their controls $[\mathrm{F}(2,18)=4.726, \mathrm{p}<0.05]$ and an increase in struggling behaviours in treated SLOW rats.

Statistical analysis of the total 10 minute test period revealed a significant strain $\mathrm{x}$ treatment interaction $[F(2,39)=3.370, p<0.05]$, where SLOW rats showed a significantly greater percentage of struggle than FAST rats. Furthermore, although the treatment effect in SLOW rats that received omega- 3 supplementation carried into the 10 minute analysis $[\mathrm{F}(2,21)=15.797$, $\mathrm{p}<0.0001]$, this effect was not present in FAST rats $[\mathrm{F}(2,18)=0.294, \mathrm{p}=0.749$, n.s.]. See Figure 9 . 


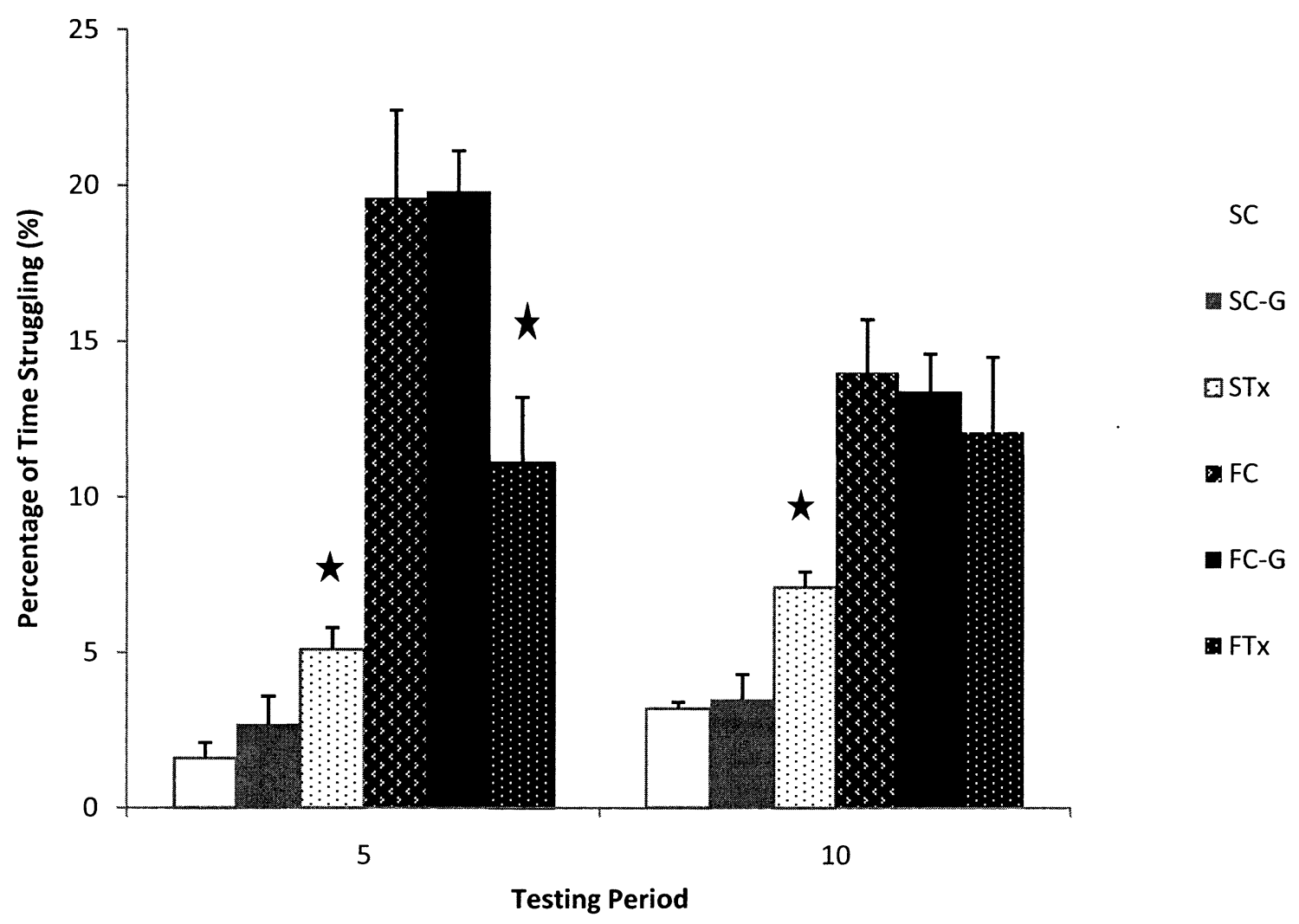

Figure 9: Relative hyperactivity/impulsivity in the restraint test. Mean (+/- S.E.M.) percentage of time spent struggling by FAST versus SLOW control and treated rats in the five and ten minute restraint tests. Significant strain difference. $P<0.05$.

* Significantly different from the two within-strain control groups 


\section{Kindling}

Measurements associated with local amygdala excitability prior to kindling

Statistical examination of ADTs within the left and right amygdalae prior to kindling did not reveal a significant main effect of $\operatorname{strain}[F(1,37)=2.361, p=0.133$, n.s. $]$ or treatment $[F(2,37)=0.197, p=0.822$, n.s. $]$, indicating that pre-kindling ADTs were similar between the strains and that neither maternal gavage nor omega-3 supplementation altered ADT intensities in either FAST or SLOW rats. See Figure 10.

As has been observed in previous research (Gilby, et al., 2009), upon initial ADT elicitation FAST rats exhibited longer mean after discharge durations (ADDs) than SLOW rats $[F(1,37)=13.740, p<0.001]$. Omega-3 supplementation, however, did not significantly affect mean ADD within $\operatorname{SLOW}[\mathrm{F}(2,21)=1.048, \mathrm{p}=0.368$, n.s. $]$ or FAST rats $(2,14)=1.861, \mathrm{p}=0.192$, n.s.]. See Figure 11.

Importantly, as is generally observed (Azarbar, et al., 2010; Gilby, et al., 2009), no significant overall correlation was evident between stimulus intensity and ADD $\left(\mathrm{r}^{2}=0.143\right)$ during ADT elicitation, suggesting relative ADDs are not affected by the intensity of the precipitating stimulus, but rather reflective of an innate resistance to seizure propagation. 


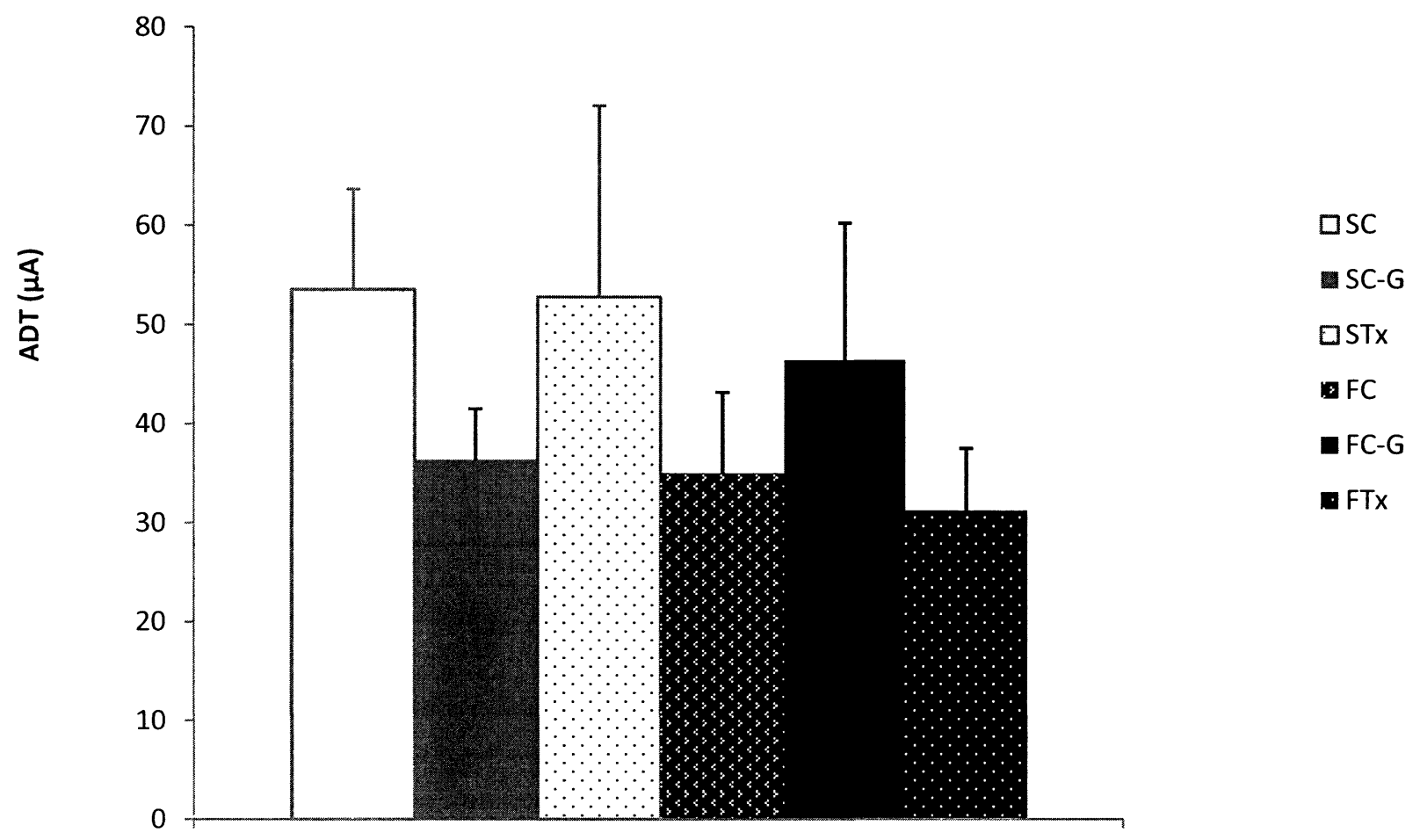

Figure 10: Mean pre-kindled ADT associated with local amygdala excitability. Mean (+/S.E.M.) pre-kindling afterdischarge threshold of FAST versus SLOW control and treated rats. 


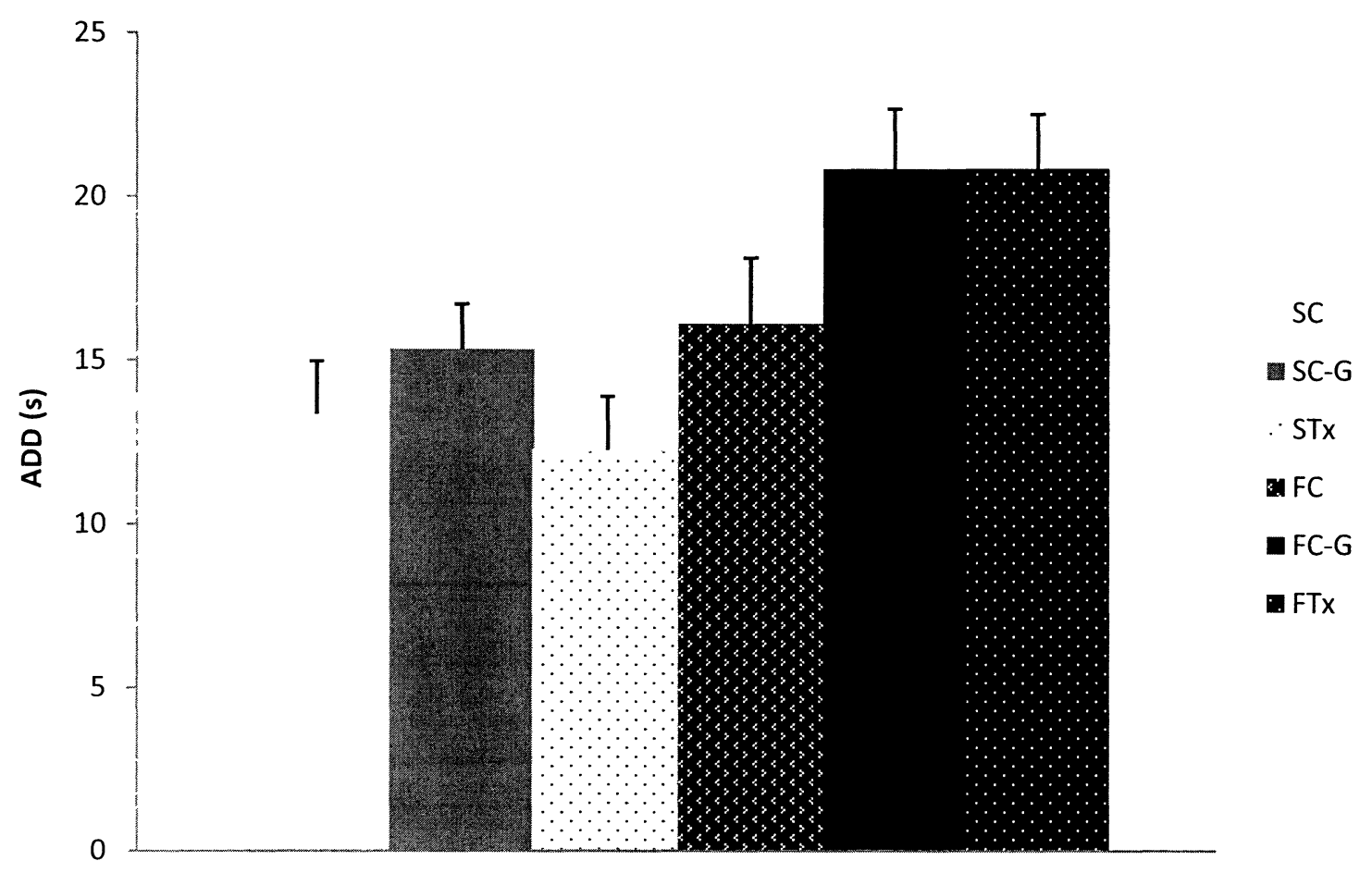

Figure 11: Mean pre-kindled ADD associated with local amygdala excitability. Mean (+/S.E.M.) pre-kindling afterdischarge duration in FAST versus SLOW control and treated rats. 


\section{Measures of epileptogenesis/neuroplasticity}

Kindling rate is an accepted measure of epileptogenic capacity and is defined as the number of once daily stimulations necessary to elicit a fully generalized (stage 5) seizure. As these strains were developed based on KR, differential rates were expected, with FAST rats kindle significantly faster than SLOW rats (Racine, et al., 1999). Analysis of KR data in this study confirmed the expected strain difference $[F(1,37)=106.528, p<0.0001]$ as well as a significant treatment effect $[\mathrm{F}(2,21)=4.608, \mathrm{p}<0.05]$ where maternal omega-3 supplementation in SLOW, but not FAST, rats led to a decrease in the number of stimulations required to elicit a stage 5 seizure compared to SLOW controls $(\mathrm{p}<0.001)$. See Figure 12.

SLOW rats typically acquire greater cumulative ADDs than FAST rats before they achieve their first stage-5 seizure (Gilby, et al., 2009), a result which was replicated in this experiment $[F(1,35)=7.22, p<0.05]$. As observed in the kindling rate analysis, omega-3 treatment affected cumulative ADDs in SLOW $(\mathrm{p}<0.01)$ rats compared to SLOW control. No treatment effect was measured in $\operatorname{FAST}[F(2,14)=0.070, p=0.932$, n.s. $]$ rats. See Figure 13 . 


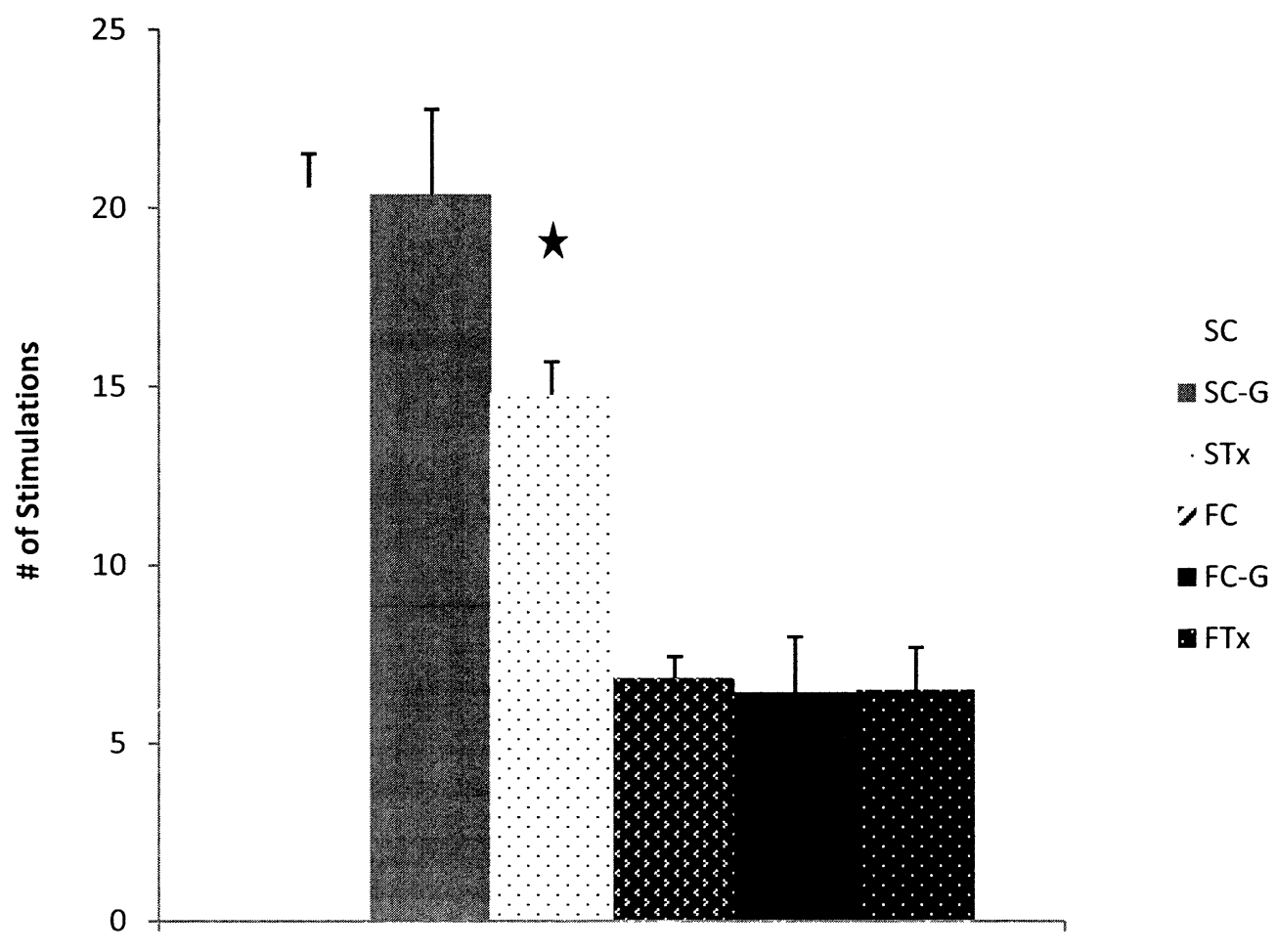

Figure 12: Mean kindling rate to the first stage-5 seizure. Mean kindling rate (+/- S.E.M.) of FAST versus SLOW control and treated rats. Significant strain difference. $P<0.05$.

$\star$ Significantly different from within-strain controls. $P<0.05$ 


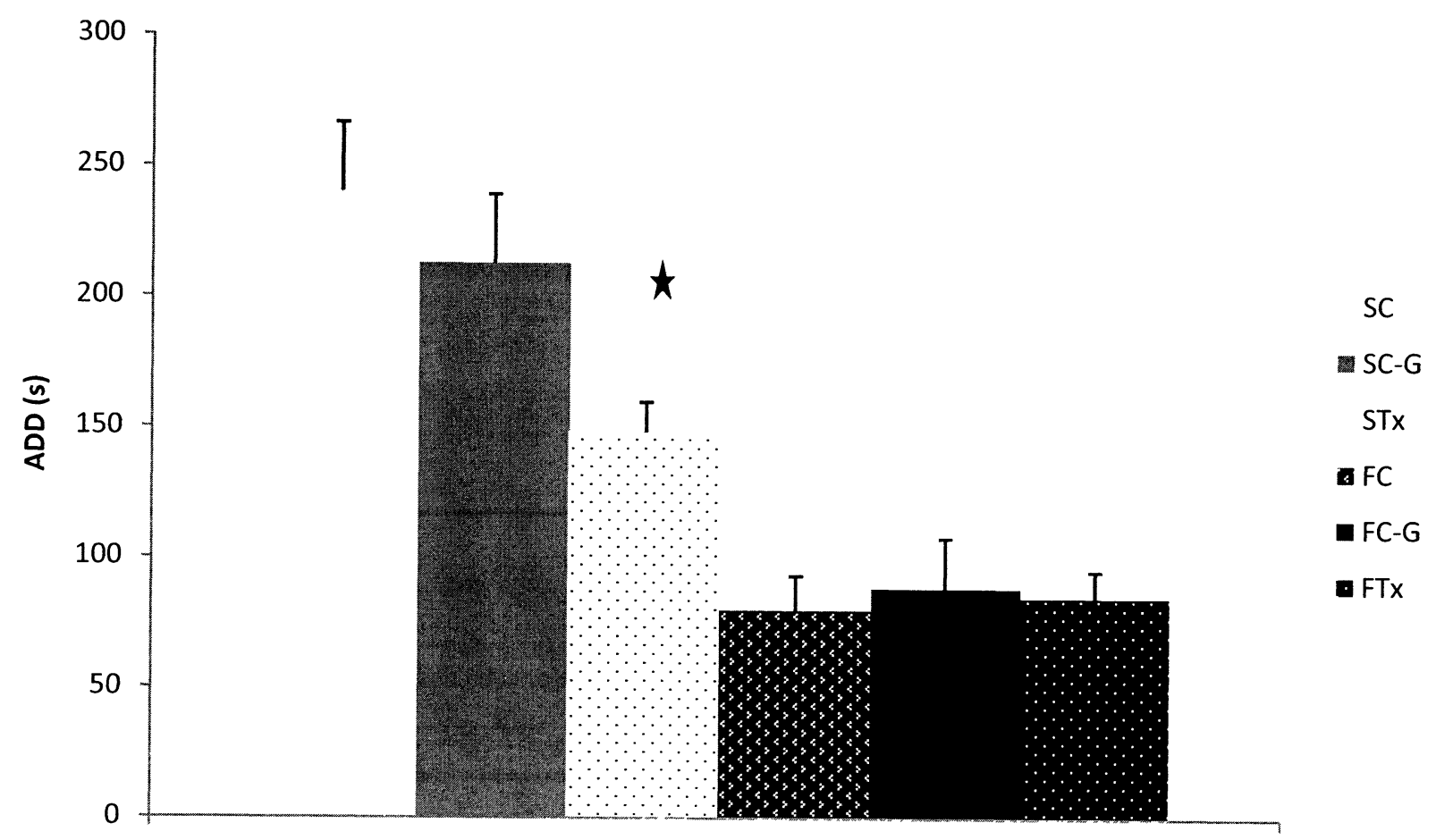

Figure 13: Mean cumulative ADD to the first stage-5 seizure. Mean Cumulative ADD (+/-

S.E.M) of FAST versus SLOW control and treated rats. Significant strain difference. $P<0.05$.

* Significantly different from within-strain control. $P<0.05$. 


\section{Generalized seizure profiles}

Once rats were fully kindled, behavioural and electrographic characteristics of six generalized stage 5 seizures were examined in order to determine if maternal omega-3 supplementation resulted in differential within-strain fully generalized seizure patterns. Specific daily measurements included latency to seizure behaviour onset, duration of partial seizure behaviour (stage 1-4), duration of fully generalized (stage 5) seizure behaviour and the total ADD associated with Stage 5 elicitation. Once kindled, typical strain differences include a decreased latency to seizure behaviour onset as well as an increased duration of partial and fully generalized seizures and ADDs in FAST versus SLOW rats (Anisman \& McIntyre, 2002; Azarbar, et al., 2010; Gilby, et al., 2009).

While analysis of latency to seizure behaviour onset did not reveal the typical strain effect $[\mathrm{F}(1,35)=1.156, \mathrm{p}=0.290 ;$ n.s. $]$, a significant main effect of procedure was identified in FAST rats $[F(2,18)=4.984, p<0.02]$ where non-gavaged control rats exhibited seizure behaviours faster than within-strain gavaged rats. No treatment/procedural effects were present within the SLOW strain $[\mathrm{F}(2,20)=0.340, \mathrm{p}=0.681)$. See Figure 14 .

As expected, typical strain differences were apparent when analyzing the mean duration of partial seizures $[F(1,38)=19.21, p<0.001]$ where FAST rats showed longer mean duration of partial seizure behaviours compared to SLOW rats. The treatment effect did not escape the procedural effect within FAST rats where non-gavaged controls had significantly higher mean durations of partial seizure behaviour $[F(2,37)=0.499, p=0.611]$ compared to gavaged FAST rats. See Figure 15.

In this study, a two way ANOVA revealed a main effect of strain where, in alignment with previous research (Gilby, et al., 2009; McIntyre \& Gilby, 2007; McIntyre, et al., 1999; 
McIntyre, et al., 2002), FAST rats showed a longer mean duration of fully generalized seizure $[F(1,38)=12.923, p<0.001]$ compared to SLOW rats.

Examination of the effect of omega-3 supplementation revealed a significant effect with regards to the duration of stage 5 seizure in $\operatorname{SLOW}$ rats $[F(2,20)=3.889, p<0.05]$, wherein seizures exhibited by SLOW rats were significantly shorter in treated rats than controls. Omega3 supplementation did not have an effect on the duration of stage- 5 seizures in FAST rats $[F(2,18)=0.642, p=0.538$, n.s. $]$. See Figure 16 .

In this experiment, analysis revealed a significant strain effect $[F(1,38)=29.49$, $\mathrm{p}<0.0001]$, where SLOW rats had significantly shorter mean ADDs compared to FAST rats. No significant effect of treatment was apparent $[\mathrm{F}(2,37)=0.700, \mathrm{p}=0.503]$, indicating maternal omega-3 supplementation did not significantly affect mean ADDs in either strains. See Figure 17. 


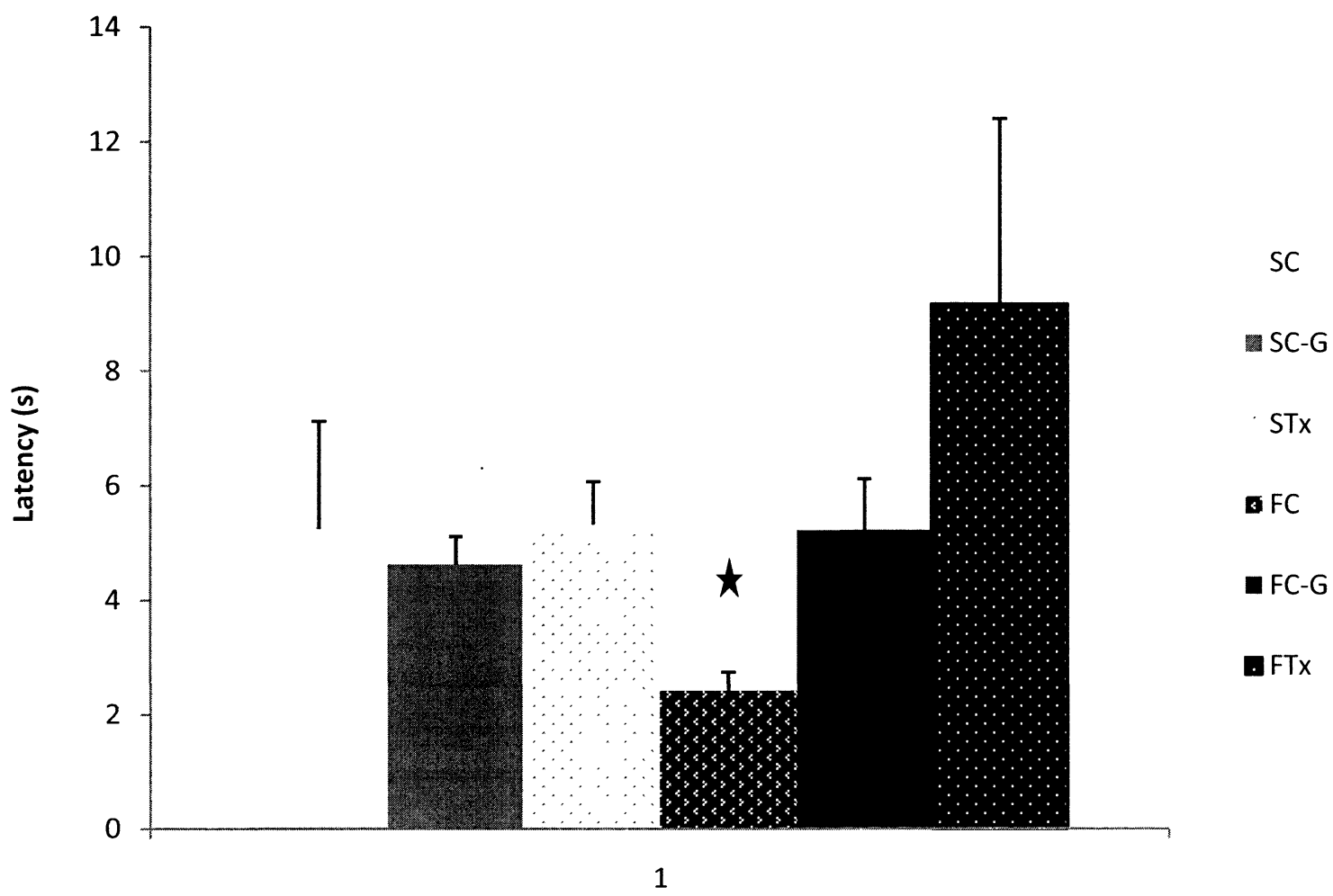

Figure 14: Mean latency to onset of partial seizure (stage 1-4) associated with elicitation of 6 stage-5 seizures. Mean latency to exhibit seizure behaviours (+/- S.E.M.) in FAST versus SLOW control and treated kindled rats.

$\star$ Significantly different from all other groups. $P<0.05$ 


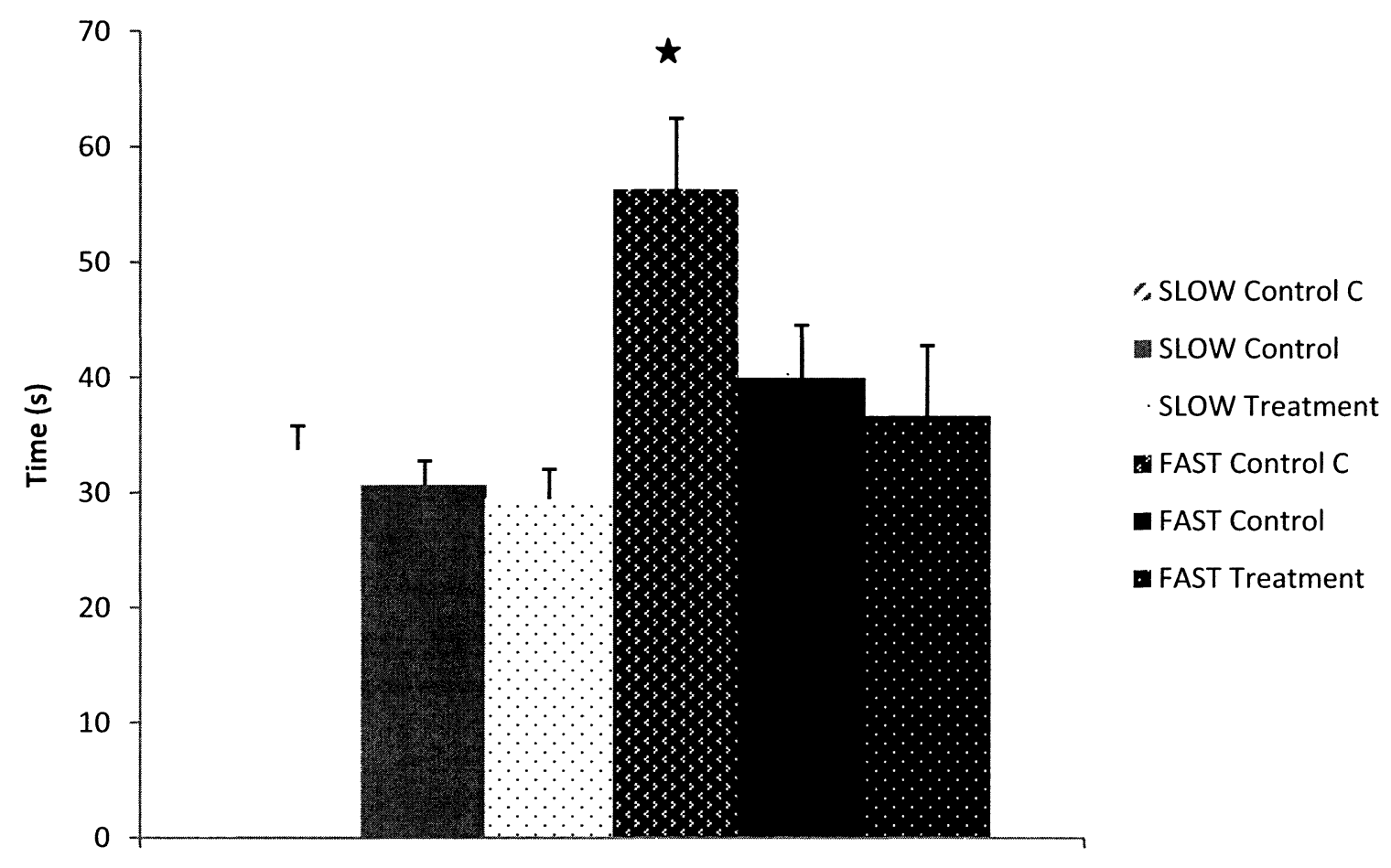

Figure 15: Mean duration of partial seizure (stage 1-4) behaviour associated with elicitation of 6 stage-5 seizure. Mean duration of partial seizure (+/- S.E.M.) in FAST versus SLOW control and treated kindled rats.

$\star$ Significantly different from within- strain gavaged groups. $P<0.05$ 


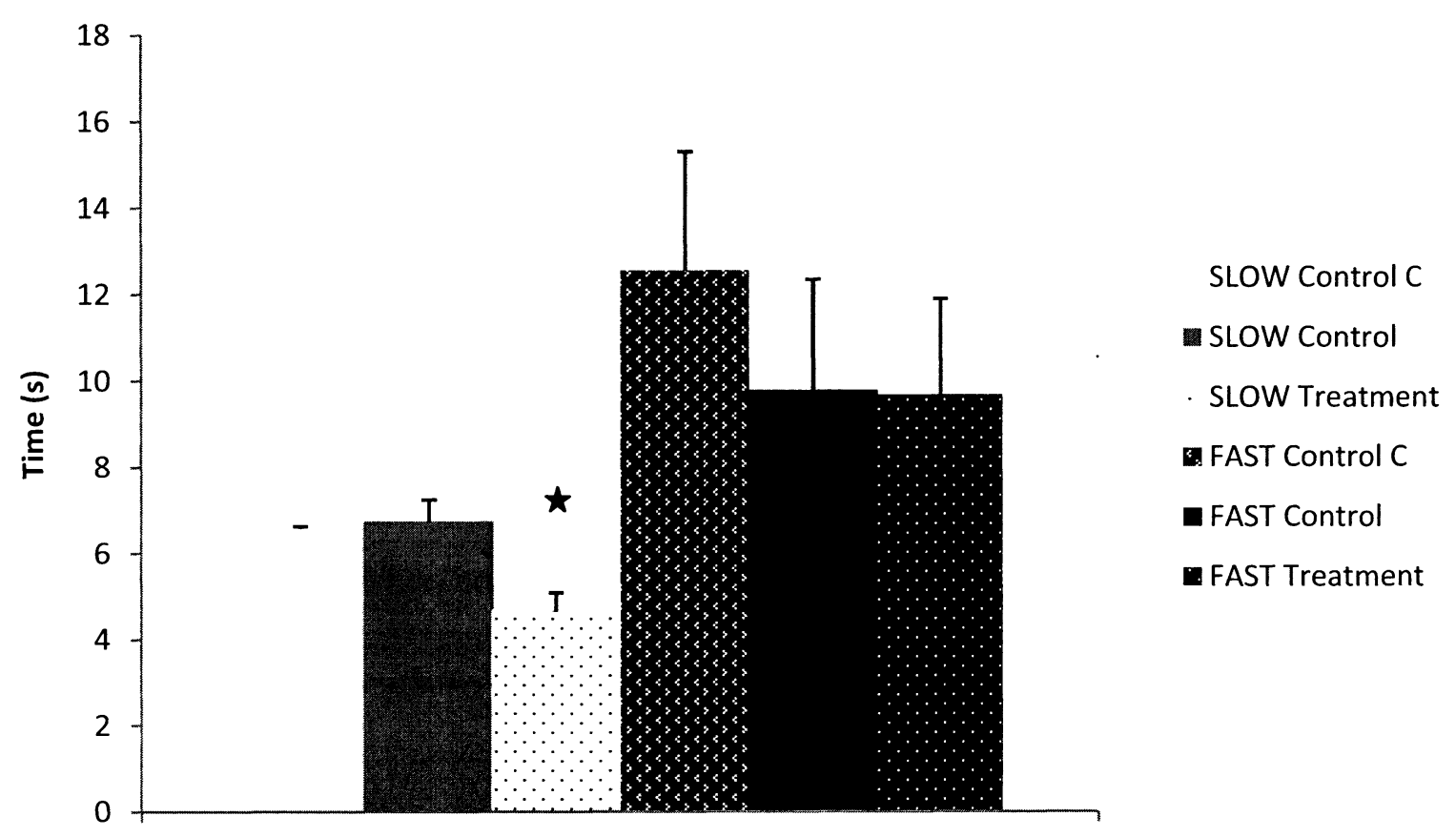

Figure 16: Mean duration of fully generalized seizure behaviour associated with elicitation of 6 successive stage-5 seizures. Mean duration of stage-5 seizure (+/- S.E.M.) in FAST versus SLOW control and treated kindled rats.

$\star$ Significantly different from within-strain controls. $P<0.05$ 


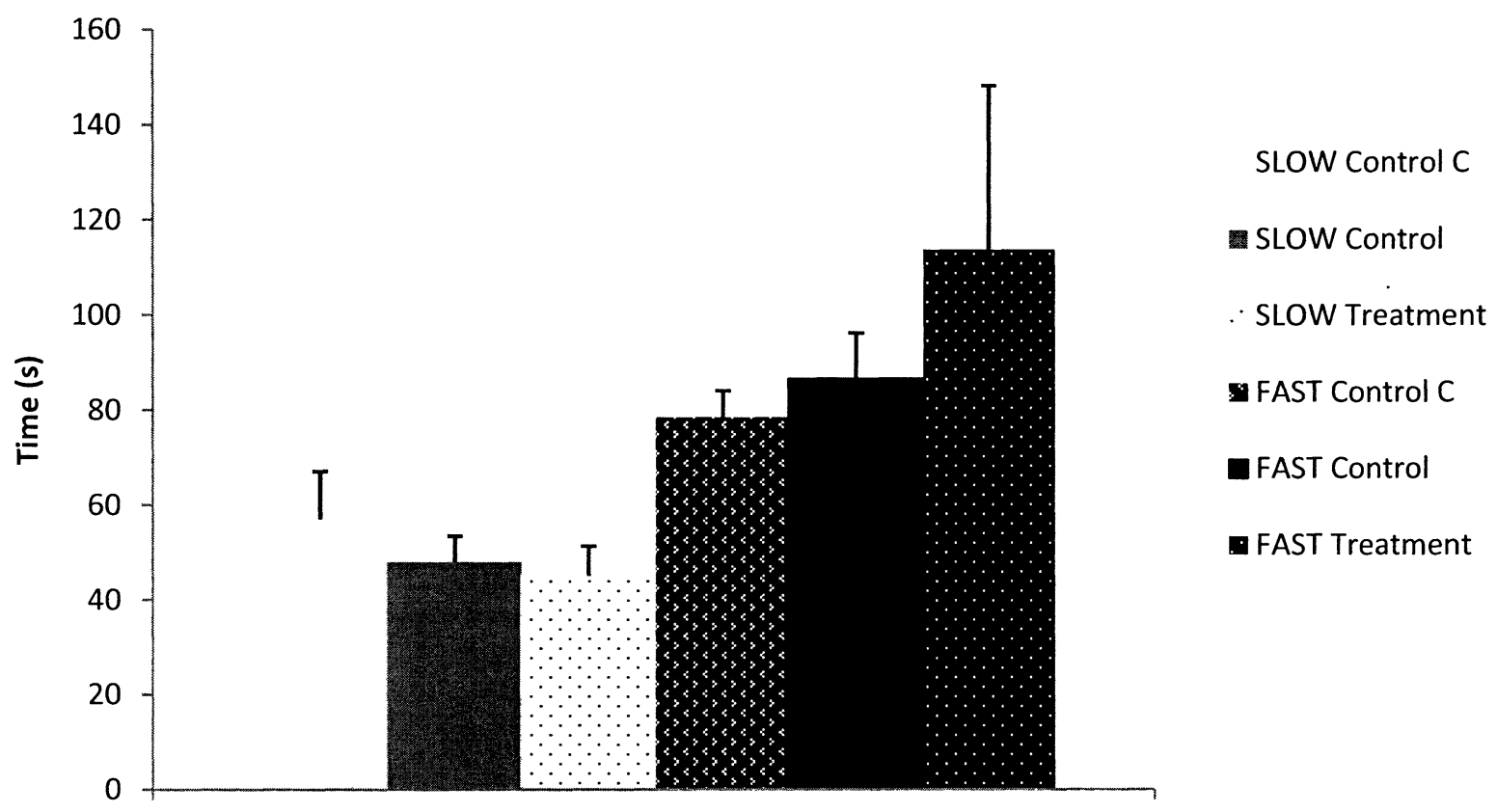

Figure 17: Mean ADD associated with elicitation of 6 successive stage-5 seizures. Mean afterdischarge duration (+/- S.E.M.) in FAST versus SLOW control and treated kindled rats. 


\section{Offspring Viability}

In order to assess the effect of maternal omega-3 fatty acid supplementation on fertility success, the percentage of successful pregnancies per group was calculated. Results indicate an astounding negative effect of omega-3 supplementation in FAST rats where offspring viability success was a mere $15 \%$, while in FAST gavaged control rats was approximately $50 \%$. Omega3 supplementation seemingly did not affect SLOW rats as both control and treated rats showed an offspring viability rate of $65 \%$. See Figure 19 . 


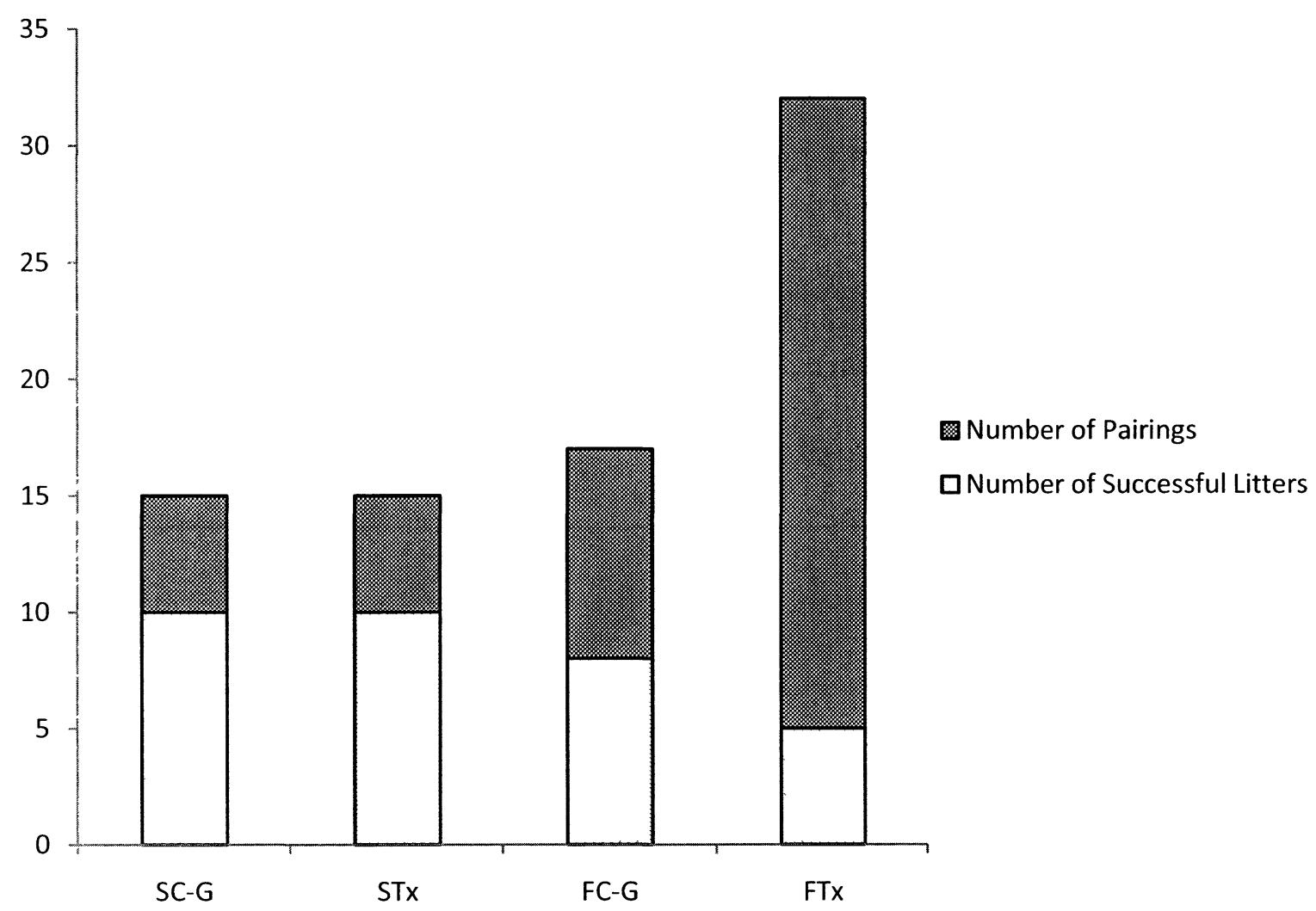

Figure 18: Mean Offspring Viability. Mean amount of successful litters in Fast versus SLOW gavaged control and treated rats. 
NEFA

FAST rats typically show reduced levels of plasma NEFA levels compared to SLOW rats (Gilby, et al., 2009). Analysis of results specific to this experiment again found significant strain differences $[\mathrm{F}(1,36)=5.289, \mathrm{p}<0.05]$ with SLOW rats showing increased NEFA levels compared to FAST rats. A significant effect of treatment $[\mathrm{F}(1,36)=6.088, \mathrm{p}<0.02]$ was also revealed where maternal omega-3 supplementation led to a reduction in measured plasma NEFA levels in both FAST $(\mathrm{p}<0.05)$ and SLOW $(\mathrm{p}<0.01)$ strains. See Figure 19. 


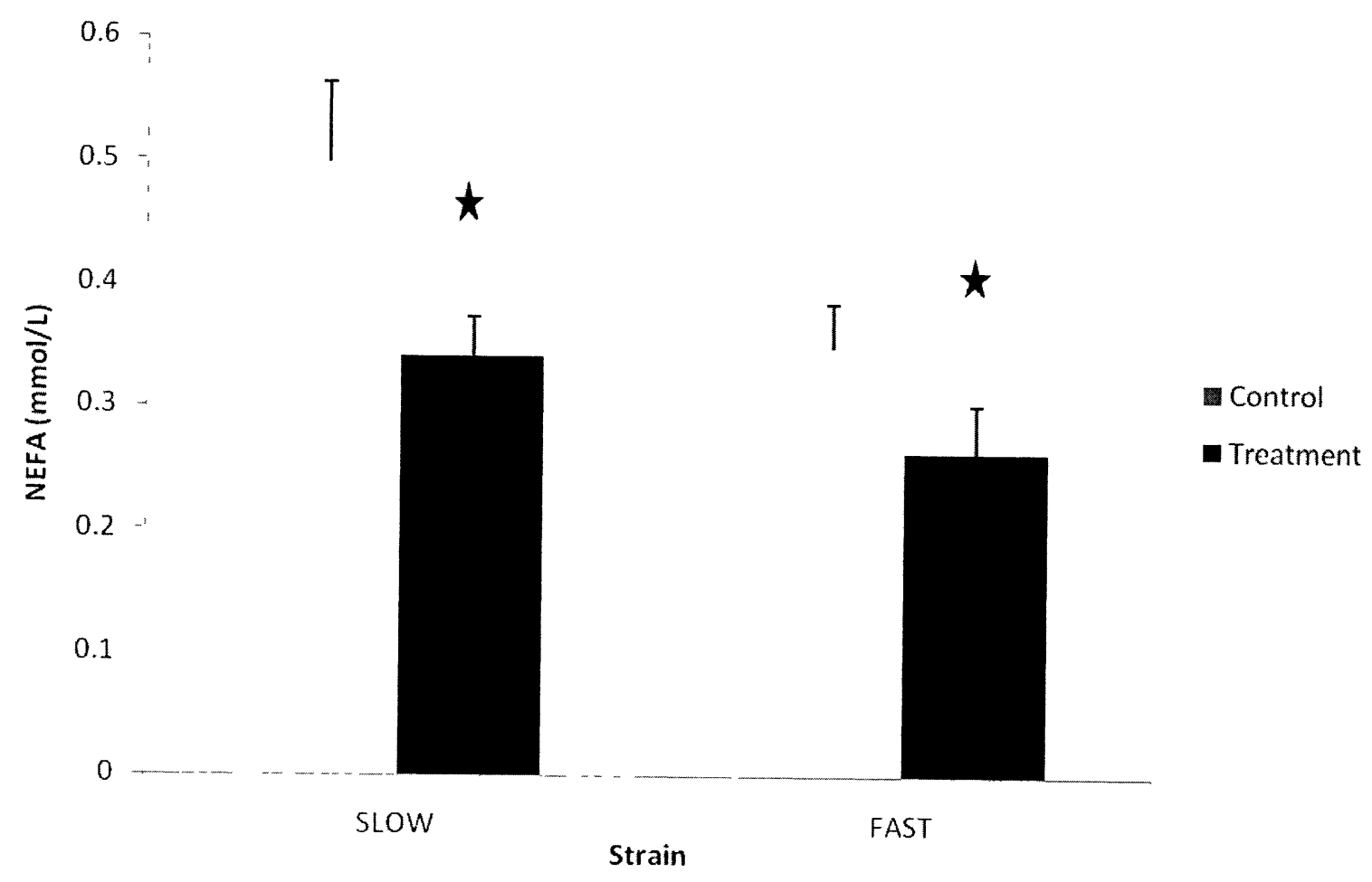

Figure 19: Mean plasma NEFA levels. Mean maternal NEFA levels (+/- S.E.M.) in Fast versus SLOW control and treated rats. Significant strain difference. $P<0.05$.

$\star$ Significantly different from within-strain controls. $P<0.05$. 


\section{Discussion}

Dietary supplementation with omega-3 FA has been shown to increase plasma omega-3 levels and simultaneously reduce negative ADHD/ASD behavioural symptomology (Joshi et al., 2006; Richardson \& Puri, 2002; Sinn \& Bryan, 2007; Sorgi, Hallowell, Hutchins, \& Sears, 2007). Positive effects of omega-3 FA supplementation have also been observed in epilepsy patients with typical results including a reduction in seizure severity, duration and frequency (Cysneiros et al., 2010; Yuen \& Sander, 2004). Indeed, FA supplementation more generally has been associated with remediation of symptoms associated with various central nervous system disorders (Genuis \& Schwalfenberg, 2006; Helland, et al., 2003; Horrobin \& Bennett, 1999; Uauy, Hoffman, Peirano, Birch, \& Birch, 2001). An extremely successful example would include the ketogenic diet (high in fats, low in carbohydrates and protein), which is rapidly becoming a treatment of choice for many epilepsy patients given its reported ability to fully arrest seizures in epilepsy patients that once averaged over 10 seizures a day in the presence of typical anticonvulsant treatments that have failed (Freeman \& Vining, 1999; Lefevre \& Aronson, 2000). Accordingly, animals consuming a ketogenic diet show a significant reduction in cerebral excitability, which contributes to reduced seizure susceptibility as measured in the kindling paradigm (de Almeida Rabello Oliveira et al., 2008; Kim do \& Rho, 2008). Although the effect of the ketogenic diet has only been rudimentarily investigated with regards to Autism, results have indicated a reduction in several Childhood Autism Rating Scale (CARS) parameters in autistic children after six months of dietary modification (Evangeliou et al., 2003). Given these and previous data generated within our own laboratory (Azarbar, et al., 2010; Gilby, Crino, et al., 2007; Gilby, et al., 2009; Gilby, Thorne, et al., 2007), it was predicted that in this study maternal omega-3 supplementation might lead to a reduction in the natural tendency towards heightened 
seizure sensitivity, hyperactivity/impulsivity and relative learning deficits in FAST rats. Effects of omega-3 supplementation were also assessed in the seizure-resistant SLOW rats, a natural comparison strain for FAST strain.

In this study, maternal omega-3 supplementation throughout gestation and lactation ultimately produced differential effects in SLOW and FAST rats. In FAST rats, it reduced measured impulsivity as well as marginally increased long term memory, whereas in SLOW rats it increased seizure susceptibility and reduced both cumulative ADDs necessary to elicit a fully generalized seizure and the duration of the fully generalized seizure. Dramatic secondary effects of maternal omega-3 supplementation included reduced offspring viability in FAST, but not SLOW rats, as well as a measured reduction in plasma NEFA levels in both strains.

\section{Pup Viability}

Breeding success and offspring viability in the laboratory can be influenced by many factors, including temperature, humidity, cage environment and stress. In this study, there was a profound and potentially novel effect of maternal omega-3 supplementation on pup viability within FAST, but not SLOW rats. Compared to gavaged FAST controls, pup viability within the FAST treated group was reduced by $35 \%$ indicating a negative effect of omega- 3

supplementation that was unrelated to the stress of maternal gavage during pregnancy and lactation. In this experiment, although fertility success was only slightly affected as measured by pups being born, within 3 days of birth fast treated pups were cannibalized by the mothers - an action which normally indicates a biological irregularity within those pups. While, as discussed, results of previous research and this experiment have both revealed omega- 3 supplementation leads to an increase in seizure susceptibility in SLOW rats. As previous research (Gilby, et al., 
2009) has also indicated this tendency of increased seizure susceptibility in FAST rats subsequent to omega-3 supplementation in adulthood, it is conceivable that maternal omega-3 supplementation actually created spontaneous, and lethal seizures in FAST offspring. Indeed spontaneous seizures in FAST offspring following low dose omega-3 supplementation in pregnant FAST and SLOW females have already been documented within our laboratory (unpublished data). If reliable, mothers with a history of epilepsy, ADHD or ASD in their family would be ill-advised to consume high doses of omega-3s during pregnancy, as it may lead to improper or even fatal neurological development in the fetus.

Plasma Non-esterified Fatty Acids (NEFA)

Previous research (Gilby, et al., 2009) indicates FAST rats have significantly lower circulating levels of plasma NEFA compared to SLOW rats. This result was replicated in the present study. Further detailed investigation has indicated FAST rats are deficient in DHA (22:6(n-3)) and DPA (22:5(n-3)) omega-3 FAs (unpublished data), which are specifically present in fish oil. As omega-3s are EFAs that must be ingested, or synthesized via elongation of precursors also obtained through the diet, these observed strain differences are of particular interest given FAST and SLOW rats were maintained on identical diets. Observed strain differences, therefore, appear to be indicative of differential lipid handling between FAST and SLOW rats. Clinically, reduced plasma omega-3 levels have been associated with the behavioural manifestation of ADHD/ASD symptomology (Antalis, et al., 2006; Biederman \& Faraone, 2005; Colter, Cutler, \& Meckling, 2008; Sinn \& Bryan, 2007) and psychiatric disorders such as depression and schizophrenia (Fenton, Dickerson, Boronow, Hibbeln, \& Knable, 2001). Although the mechanism relating behavioural symptomology observed in these disorders and 
reduced omega-3 FA levels is not known at this time, it continues to be a topic of significant interest within these fields of research.

As observed in this and previous experiments (Gilby, et al., 2009), omega-3

supplementation leads to a decrease in overall NEFA levels. Although initially counter intuitive, this decrease in NEFA levels associated with supplementation is likely due to an overall reduction in biosynthesis of FA, as they are readily available due to supplementation (Lee \& Lip, 2003). Secondary research investigating this phenomenon suggests reduced NEFA levels are associated with increased $\beta$-oxidation or inhibition of triglyceride-synthesizing enzymes (Harris \& Bulchandani, 2006). With regards to clinical implications, these results indicate although omega-3 supplementation may be successful in reducing ADHD type behaviour, long term supplementation may introduce the possibility of negative biochemical consequences. As omega-3 supplementation research in relatively novel, the long term effects of such a treatment are unclear. On the other hand, because the treatment has been shown to have important consequences for the patients in a variety of contexts, it will certainly continue to be a highly investigated area of behavioural neuroscience.

\section{Behaviour}

While typical strain differences were revealed through OF testing, maternal omega-3 supplementation did not affect measured hyperactivity in either FAST or SLOW rats. The lack of treatment effect on relative hyperactivity in FAST rats was unexpected, however, is in accordance with previous published data using omega-3 to treat these strains as adults (Gilby, et al., 2009). Clinical research investigating the efficacy of omega-3 supplementation on ADHDassociated hyperactivity in children, in fact, has produced mixed results (Chalon, 2009; Raz \& 
Gabis, 2009). The reason for this is unclear, but is most likely owing to the vast differences in experimental design employed in those studies, including mode of administration, duration of supplementation, and the outcome measures evaluated. Thus, no clear clinical relevance can be extrapolated from our lack of effect on hyperactive behaviours in the FAST rats.

The MWM is a task generally used in animal modeling to provide information relevant to the animal's capacity for learning and memory, particularly spatial short (STM) and long term memory (LTM). Characteristic strain differences with SLOW rats showing increased STM and LTM compared to FAST rats was observed in this study. Furthermore, maternal omega-3 supplementation significantly enhanced the performance of FAST rats on the fourth day of testing indicating supplementation did lead to some improvement in the FAST strain relative to their within-strain controls. This significant effect of treatment is interesting considering previous research involving direct supplementation in adults (Gilby, et al., 2009) did not report any benefit for the FAST rats in the MWM from omega-3 supplementation. It may well be relevant, however, that the version of MWM utilised in this study, and in Gilby (2009), is the most simple variant of this task (no distracting cues, platform remained in the same location throughout the experiment, etc.). Thus, it is conceivable that the benefits of omega-3 supplementation may have greater detectability when more difficult MWM variants are employed. Certainly, a vast array of clinical research has confirmed reduced memory performance in individuals diagnosed with ADHD/ASD compared to healthy individuals (Liu \& Wang, 2002), and a small study by Richardson (2006) reported increased STM in patients that received omega-3 supplementation. Experimentation involving the use of omega-3 supplementation as a method of treatment/prevention of ADHD-type behavioural characteristics 
is still in its infancy, and thus, any discrepancies between laboratory and clinical studies on this topic will be of the fodder of future research.

Using the restraint task as a behavioural gauge, maternal omega-3 supplementation reduced struggling behaviours in FAST rats, while it increased struggling in SLOW rat. Although observed behaviour in FAST rats was akin to results of previous research (Gilby, et al., 2009), the increase in SLOW behaviour was puzzling. Important to note, however, strain typical differences were still measurable with FAST rats exhibiting significantly greater struggling behaviours than SLOW rats. With respect to the effects in FAST rats, similar results have been shown in the clinical conditions that the FAST rats model, where omega-3 supplementation led to a reduction in impulsive-like behaviours in highly impulsive ADHD/ASD type individuals (Schachter et al., 2005). The reason omega-3 supplementation increased struggling behaviour in the SLOW rats is difficult to determine, and may relate to the kindling results discussed below. Indeed, it may be reflective of a normalizing effect of omega-3 supplementation, as SLOW rats are not to be confused with 'normal' outbred rat strains. It is also noteworthy that both the open field and restraint testing paradigms can provide information relevant to the relative tendency of an animal towards hyperactivity. The fact that maternal omega-3 treatment altered FAST and SLOW behaviour in the restraint task, but not in the open field clearly demonstrates that behavioural assessments using these two measures are not interchangeable or isomorphic. Restraint activity is not merely another measure of hyperactivity. It is suggested, therefore, that the measured differences between restraint and OF performances highlight impulsivity in the restraint paradigm. 


\section{Kindling}

In the kindling model of epilepsy, the primary measure of interest is the kindling rate (KR). As revealed in this study, FAST rats typically exhibit faster KRs than SLOW rats, thus indicating they are more seizure-prone than SLOW rats (Gilby, Crino, et al., 2007; Gilby, et al., 2009; McIntyre, et al., 1999; McIntyre, et al., 2002). Analysing treatment effects in this context indicated that maternal omega-3 supplementation increased relative seizure sensitivity in SLOW but not FAST rats, as evidenced by the significant reduction in KR compared to their within strain control. Indeed, it may appear that increased seizure sensitivity following maternal omega-3 supplementation in SLOW but not FAST rats is paradoxical, however, the lack of measured treatment effect in FAST rats may be due to the inability of seizure sensitivity to increase due to its already heightened levels in these animals (i.e., a 'floor effect'). These results are in line with previous research using omega-3 treatment in these strains (Gilby, et al., 2009).

Typically FAST rats exhibit higher seizure sensitivity than SLOW rats as expressed by longer afterdischarge durations (ADD) resulting from a single stimulation, thus indicating they are also less capable of shutting down post- stimulation epileptic activity than SLOW rats (Gilby, et al., 2009; McIntyre, et al., 2002). In this experiment, maternal omega-3 supplementation did not significantly alter strain-specific ADDs at the ADT indicating this treatment may be effective in enhancing epileptogenic capacity (KR) but does not alter the brain's capacity to terminate or arrest electrographic seizures.

Examination of the generalized convulsive stage- 5 seizure profile revealed typical strain differences with regards to all measures taken except for latency to onset of partial seizures. Although in association with their increased seizure susceptibility FAST rats typically exhibit 
seizure behaviours more quickly than SLOW rats (Gilby, et al., 2009), the lack of strain difference may be due to the relatively quick onset of behaviour expressed by all animals. Further examination revealed maternal omega-3 supplementation did not significantly alter the latency to onset or the duration of partial seizures in SLOW rats, however, it did significantly reduce the duration of fully generalized stage- 5 seizures. Thus the treatment exacerbated the KR or spread of seizures in the SLOW rats but facilitate the truncation or shortening of the seizure. This result suggests that the mechanisms of seizure spread and those of seizure arrest are likely independent.

Gavage

A potential concern involving the experimental design in this study included the use of gavage as the method of omega- 3 supplementation administration. Humans would typically receive omega-3 supplementation orally in pill form and, although gavage has been observed to negatively impact experimental results due to increased animal stress (Balcombe, Barnard, \& Sandusky, 2004; de Meijer, Le, Meisel, \& Puder, 2010), the desire here was to supplement animals in a way that would mimic the clinical route of administration and ensure dosing remained accurate and consistent. As previously mentioned in the Methodology section, in order to ensure any observed effects of omega-3 supplementation were not confounded by the negative effects of gavage, the behaviour and kindling data of gavage per se was assessed. Importantly, the procedural effects of gavage were generally not found to significantly affect outcome measures assessed in this experiment. One exception to this was the latency to onset of behavioural seizures and the duration of partial (stage 1-4) seizure behaviours in fully kindled FAST rats. Specifically, non-gavaged FAST control rats required significantly less time to begin a convulsive seizure and had longer seizures than all other groups. As these two measures are 
directly related, this result indicates that gavage induced stressed within FAST mothers may significantly reduce seizure susceptibility in offspring. These measured differences, however, were not paired with differences in pre-kindled measures (KR, mean or cumulative ADD), suggesting gavage-induced stress may only affect specific aspects of the kindling phenomenon in fully kindled rats.

\section{Conclusion}

Multiple experiments indicate beneficial effects of omega-3 FA supplementation as a treatment of clinical disorders such as ADHD/ASD when used individually or in conjunction with pharmaceutical treatments (Chalon, 2009; Johnson, et al., 2009; Raz \& Gabis, 2009). However, the results of the present study indicate the additional possible harmful effects of such treatments, both in FAST and SLOW rats, independent of seizure sensitivity predisposition. In those with a low seizure sensitivity (SLOW rats), maternal omega-3 supplementation increased seizure sensitivity yet truncated seizure durations, and in those with a high seizure sensitivity (FAST rats) maternal omega-3 supplementation altered fetal development in such a way that it greatly reduced offspring viability. Positive results with regards to this experiment include an increase in learning and memory as well as a decrease in impulsive behaviours in FAST rats, indicating omega-3 FA alters $\mathrm{ADHD} / \mathrm{ASD}$-like behaviours in seizure prone rats as predicted from the human data.

\section{Future Directions}

Given the findings of this study, a primary research objective will be to establish the mechanism responsible for strain differences in lipid handling. Molecular research has indicated FAST rats are capable of producing essential fatty acids from their precursors, however, the rats 
problem may lie in brain/blood level imbalances of FAs. Recent results analyzed subsequent to the commencement of this experiment have also indicated FAST rats show deficits in polyunsaturated omega- 6 fatty acids, thereby suggesting behavioural and kindling differences between strains may not only be due to omega-3 fatty acid differences, but rather wide spread metabolic irregularities. All of these metabolic venues will be addressed in future studies. The ultimate goal of these studies with the strains is to understand the manner in which lipid handling impacts different individuals in a variety of behavioural contexts. Our view is that highly specific individual diagnosis will be the way of the future in medicine, where each person will be screened for their own unique metabolic profile and will receive treatments appropriate to their exact condition, a condition that certainly will be the negative mirror image of some other individual, paralleling our findings with the FAST and SLOW strains. 


\section{REFERENCES:}

Agale, S., Kulkarni, A., Ranjekar, P., \& Joshi, S. (2010). Maternal caloric restriction spares fetal brain polyunsaturated fatty acids in Wistar rats. Brain Dev, 32(2), 123-129.

Amminger, G. P., Berger, G. E., Schafer, M. R., Klier, C., Friedrich, M. H., \& Feucht, M. (2007). Omega-3 fatty acids supplementation in children with autism: a double-blind randomized, placebo-controlled pilot study. Biol Psychiatry, 61(4), 551-553.

Anisman, H., \& McIntyre, D. C. (2002). Conceptual, spatial, and cue learning in the Morris water maze in fast or slow kindling rats: attention deficit comorbidity. J Neurosci, 22(17), 7809-7817.

Antalis, C. J., Stevens, L. J., Campbell, M., Pazdro, R., Ericson, K., \& Burgess, J. R. (2006). Omega-3 fatty acid status in attention-deficit/hyperactivity disorder. Prostaglandins Leukot Essent Fatty Acids, 75(4-5), 299-308.

Azarbar, A., McIntyre, D. C., \& Gilby, K. L. (2010). Caloric restriction alters seizure disposition and behavioral profiles in seizure-prone (fast) versus seizure-resistant (slow) rats. Behav Neurosci, 124(1), 106-114.

Balcombe, J. P., Barnard, N. D., \& Sandusky, C. (2004). Laboratory routines cause animal stress. Contemp Top Lab Anim Sci, 43(6), 42-51.

Ballard, S., Bolan, M., Burton, M., Snyder, S., Pasterczyk-Seabolt, C., \& Martin, D. (1997). The neurological basis of attention deficit hyperactivity disorder. Adolescence, 32(128), 855862.

Biederman, J., \& Faraone, S. V. (2005). Attention-deficit hyperactivity disorder. Lancet, $366(9481), 237-248$. 
Boeck, C. R., Marques, V. B., Valvassori, S. S., Constantino, L. C., Rosa, D. V., Lima, F. F., et al. (2009). Early long-term exposure with caffeine induces cross-sensitization to methylphenidate with involvement of DARPP-32 in adulthood of rats. Neurochem Int, $55(5), 318-322$.

Brookes, K. J., Chen, W., Xu, X., Taylor, E., \& Asherson, P. (2006). Association of fatty acid desaturase genes with attention-deficit/hyperactivity disorder. Biol Psychiatry, 60(10), 1053-1061.

Cetin, I., Alvino, G., \& Cardellicchio, M. (2009). Long chain fatty acids and dietary fats in fetal nutrition. J Physiol, 587(Pt 14), 3441-3451.

Chalon, S. (2009). The role of fatty acids in the treatment of ADHD. Neuropharmacology, 57(78), 636-639.

Church, M. W., Jen, K. L., Jackson, D. A., Adams, B. R., \& Hotra, J. W. (2009). Abnormal neurological responses in young adult offspring caused by excess omega-3 fatty acid (fish oil) consumption by the mother during pregnancy and lactation. Neurotoxicol Teratol, $31(1), 26-33$.

Clark-Taylor, T., \& Clark-Taylor, B. E. (2004). Is autism a disorder of fatty acid metabolism? Possible dysfunction of mitochondrial beta-oxidation by long chain acyl-CoA dehydrogenase. Med Hypotheses, 62(6), 970-975.

Colter, A. L., Cutler, C., \& Meckling, K. A. (2008). Fatty acid status and behavioural symptoms of attention deficit hyperactivity disorder in adolescents: a case-control study. Nutr J, 7, 8. 
Cysneiros, R. M., Ferrari, D., Arida, R. M., Terra, V. C., de Almeida, A. C., Cavalheiro, E. A., et al. (2010). Qualitative analysis of hippocampal plastic changes in rats with epilepsy supplemented with oral omega-3 fatty acids. Epilepsy Behav, 17(1), 33-38.

Cysneiros, R. M., Terra, V. C., Machado, H. R., Arida, R. M., Albuquerque, M., Scorza, C. A., et al. (2009). Epilepsy and sudden unexpected death in epilepsy?: eat more fish! A group hypothesis. Arq Neuropsiquiatr, 67(3B), 927-929.

Davis, S. M., Katusic, S. K., Barbaresi, W. J., Killian, J., Weaver, A. L., Ottman, R., et al. (2010). Epilepsy in children with attention-deficit/hyperactivity disorder. Pediatr Neurol, $42(5), 325-330$.

de Almeida Rabello Oliveira, M., da Rocha Ataide, T., de Oliveira, S. L., de Melo Lucena, A. L., de Lira, C. E., Soares, A. A., et al. (2008). Effects of short-term and long-term treatment with medium- and long-chain triglycerides ketogenic diet on cortical spreading depression in young rats. Neurosci Lett, 434(1), 66-70.

de Meijer, V. E., Le, H. D., Meisel, J. A., \& Puder, M. (2010). Repetitive orogastric gavage affects the phenotype of diet-induced obese mice. Physiol Behav, 100(4), 387-393.

Decsi, T., \& Koletzko, B. (2005). N-3 fatty acids and pregnancy outcomes. Curr Opin Clin Nutr Metab Care, 8(2), 161-166.

DeGiorgio, C. M., Miller, P., Meymandi, S., \& Gornbein, J. A. (2008). n-3 fatty acids (fish oil) for epilepsy, cardiac risk factors, and risk of SUDEP: clues from a pilot, double-blind, exploratory study. Epilepsy Behav, 13(4), 681-684.

Donahue, S. M., Rifas-Shiman, S. L., Olsen, S. F., Gold, D. R., Gillman, M. W., \& Oken, E. (2009). Associations of maternal prenatal dietary intake of n-3 and n-6 fatty acids with 
maternal and umbilical cord blood levels. Prostaglandins Leukot Essent Fatty Acids, $80(5-6), 289-296$.

Dunstan, J. A., Mitoulas, L. R., Dixon, G., Doherty, D. A., Hartmann, P. E., Simmer, K., et al. (2007). The effects of fish oil supplementation in pregnancy on breast milk fatty acid composition over the course of lactation: a randomized controlled trial. Pediatr Res, 62(6), 689-694.

Dunstan, J. A., Mori, T. A., Barden, A., Beilin, L. J., Taylor, A. L., Holt, P. G., et al. (2003). Fish oil supplementation in pregnancy modifies neonatal allergen-specific immune responses and clinical outcomes in infants at high risk of atopy: a randomized, controlled trial. $J$ Allergy Clin Immunol, 112(6), 1178-1184.

Evangeliou, A., Vlachonikolis, I., Mihailidou, H., Spilioti, M., Skarpalezou, A., Makaronas, N., et al. (2003). Application of a ketogenic diet in children with autistic behavior: pilot study. J Child Neurol, 18(2), 113-118.

Faraone, S. V., \& Biederman, J. (1994). Is attention deficit hyperactivity disorder familial? Harv Rev Psychiatry, 1(5), 271-287.

Fenton, W. S., Dickerson, F., Boronow, J., Hibbeln, J. R., \& Knable, M. (2001). A placebocontrolled trial of omega-3 fatty acid (ethyl eicosapentaenoic acid) supplementation for residual symptoms and cognitive impairment in schizophrenia. Am J Psychiatry, 158(12), 2071-2074.

Ferrari, D., Cysneiros, R. M., Scorza, C. A., Arida, R. M., Cavalheiro, E. A., de Almeida, A. C., et al. (2008). Neuroprotective activity of omega-3 fatty acids against epilepsy-induced hippocampal damage: Quantification with immunohistochemical for calcium-binding proteins. Epilepsy Behav, 13(1), 36-42. 
Freeman, J. M., \& Vining, E. P. (1999). Seizures decrease rapidly after fasting: preliminary studies of the ketogenic diet. Arch Pediatr Adolesc Med, 153(9), 946-949.

Freeman, J. M., Vining, E. P., Pillas, D. J., Pyzik, P. L., Casey, J. C., \& Kelly, L. M. (1998). The efficacy of the ketogenic diet-1998: a prospective evaluation of intervention in 150 children. Pediatrics, 102(6), 1358-1363.

Gabis, L., Pomeroy, J., \& Andriola, M. R. (2005). Autism and epilepsy: cause, consequence, comorbidity, or coincidence? Epilepsy Behav, 7(4), 652-656.

Genuis, S. J., \& Schwalfenberg, G. K. (2006). Time for an oil check: the role of essential omega3 fatty acids in maternal and pediatric health. J Perinatol, 26(6), 359-365.

Gilby, K. L., Crino, P., \& McIntyre, D. C. (2007). Neurodevelopment in seizure-prone and seizure-resistant rat strains: recognizing conflicts in management. Epilepsia, 48 Suppl 5, 114-118.

Gilby, K. L., Jans, J., \& McIntyre, D. C. (2009). Chronic omega-3 supplementation in seizureprone versus seizure-resistant rat strains: a cautionary tale. Neuroscience, 163(3), 750758.

Gilby, K. L., Thorne, V., Patey, A., \& McIntyre, D. C. (2007). Ruling out postnatal origins to attention-deficit/hyperactivity disorder (ADHD)-like behaviors in a seizure-prone rat strain. Behav Neurosci, 121(2), 370-379.

Gochfeld, M., \& Burger, J. (2005). Good fish/bad fish: a composite benefit-risk by dose curve. Neurotoxicology, 26(4), 511-520.

Goddard, G. V., \& Douglas, R. M. (1975). Does the engram of kindling model the engram of normal long term memory? Can J Neurol Sci, 2(4), 385-394. 
Hanebutt, F. L., Demmelmair, H., Schiessl, B., Larque, E., \& Koletzko, B. (2008). Long-chain polyunsaturated fatty acid (LC-PUFA) transfer across the placenta. Clin Nutr, 27(5), 685693.

Harris, W. S., \& Bulchandani, D. (2006). Why do omega-3 fatty acids lower serum triglycerides? Curr Opin Lipidol, 17(4), 387-393.

Harris, W. S., Connor, W. E., \& Lindsey, S. (1984). Will dietary omega-3 fatty acids change the composition of human milk? Am J Clin Nutr, 40(4), 780-785.

Helland, I. B., Smith, L., Saarem, K., Saugstad, O. D., \& Drevon, C. A. (2003). Maternal supplementation with very-long-chain n-3 fatty acids during pregnancy and lactation augments children's IQ at 4 years of age. Pediatrics, 111(1), e39-44.

Hesdorffer, D. C., Ludvigsson, P., Olafsson, E., Gudmundsson, G., Kjartansson, O., \& Hauser, W. A. (2004). ADHD as a risk factor for incident unprovoked seizures and epilepsy in children. Arch Gen Psychiatry, 61(7), 731-736.

Horrobin, D. F., \& Bennett, C. N. (1999). Depression and bipolar disorder: relationships to impaired fatty acid and phospholipid metabolism and to diabetes, cardiovascular disease, immunological abnormalities, cancer, ageing and osteoporosis. Possible candidate genes. Prostaglandins Leukot Essent Fatty Acids, 60(4), 217-234.

Innis, S. M. (2007). Dietary (n-3) fatty acids and brain development. J Nutr, 137(4), 855-859.

Jen, K. L., Church, M. W., Wang, C., Moghaddam, M., Dowhan, L., Laja, F., et al. (2009). Perinatal n-3 fatty acid imbalance affects fatty acid composition in rat offspring. Physiol Behav, 98(1-2), 17-24. 
Johnson, M., Ostlund, S., Fransson, G., Kadesjo, B., \& Gillberg, C. (2009). Omega-3/omega-6 fatty acids for attention deficit hyperactivity disorder: a randomized placebo-controlled trial in children and adolescents. J Atten Disord, 12(5), 394-401.

Joshi, K., Lad, S., Kale, M., Patwardhan, B., Mahadik, S. P., Patni, B., et al. (2006). Supplementation with flax oil and vitamin C improves the outcome of Attention Deficit Hyperactivity Disorder (ADHD). Prostaglandins Leukot Essent Fatty Acids, 74(1), 1721.

Kaufmann, R., Goldberg-Stern, H., \& Shuper, A. (2009). Attention-deficit disorders and epilepsy in childhood: incidence, causative relations and treatment possibilities. J Child Neurol, 24(6), 727-733.

Kim do, Y., \& Rho, J. M. (2008). The ketogenic diet and epilepsy. Curr Opin Clin Nutr Metab Care, 11(2), 113-120.

Lalonde, R., \& Strazielle, C. (2009). The relation between open-field and emergence tests in a hyperactive mouse model. Neuropharmacology, 57(7-8), 722-724.

Lee, K. W., \& Lip, G. Y. (2003). The role of omega-3 fatty acids in the secondary prevention of cardiovascular disease. QJM, 96(7), 465-480.

Lefevre, F., \& Aronson, N. (2000). Ketogenic diet for the treatment of refractory epilepsy in children: A systematic review of efficacy. Pediatrics, 105(4), E46.

Liu, Y., \& Wang, Y. (2002). [Cognitive functions of children with attention deficit/hyperactivity disorder]. Zhonghua Yi Xue Za Zhi, 82(6), 389-392.

Makrides, M., Gibson, R. A., McPhee, A. J., Collins, C. T., Davis, P. G., Doyle, L. W., et al. (2009). Neurodevelopmental outcomes of preterm infants fed high-dose docosahexaenoic acid: a randomized controlled trial. JAMA, 301(2), 175-182. 
McIntyre, D. C., \& Gilby, K. L. (2007). Genetically seizure-prone or seizure-resistant phenotypes and their associated behavioral comorbidities. Epilepsia, 48 Suppl 9, 30-32.

McIntyre, D. C., Kelly, M. E., \& Dufresne, C. (1999). FAST and SLOW amygdala kindling rat strains: comparison of amygdala, hippocampal, piriform and perirhinal cortex kindling. Epilepsy Res, 35(3), 197-209.

McIntyre, D. C., Poulter, M. O., \& Gilby, K. (2002). Kindling: some old and some new. Epilepsy Res, 50(1-2), 79-92.

McNamara, R. K., \& Carlson, S. E. (2006). Role of omega-3 fatty acids in brain development and function: potential implications for the pathogenesis and prevention of psychopathology. Prostaglandins Leukot Essent Fatty Acids, 75(4-5), 329-349.

Mohapel, P., \& McIntyre, D. C. (1998). Amygdala kindling-resistant (SLOW) or -prone (FAST) rat strains show differential fear responses. Behav Neurosci, 112(6), 1402-1413.

Murphy, P., Likhodii, S. S., Hatamian, M., \& McIntyre Burnham, W. (2005). Effect of the ketogenic diet on the activity level of Wistar rats. Pediatr Res, 57(3), 353-357.

Racine, R. J., Steingart, M., \& McIntyre, D. C. (1999). Development of kindling-prone and kindling-resistant rats: selective breeding and electrophysiological studies. Epilepsy Res, 35(3), 183-195.

Raz, R., \& Gabis, L. (2009). Essential fatty acids and attention-deficit-hyperactivity disorder: a systematic review. Dev Med Child Neurol, 51(8), 580-592.

Richardson, A. J. (2006). Omega-3 fatty acids in ADHD and related neurodevelopmental disorders. Int Rev Psychiatry, 18(2), 155-172.

Richardson, A. J., \& Puri, B. K. (2002). A randomized double-blind, placebo-controlled study of the effects of supplementation with highly unsaturated fatty acids on ADHD-related 
symptoms in children with specific learning difficulties. Prog Neuropsychopharmacol Biol Psychiatry, 26(2), 233-239.

Richardson, A. J., \& Ross, M. A. (2000). Fatty acid metabolism in neurodevelopmental disorder: a new perspective on associations between attention-deficit/hyperactivity disorder, dyslexia, dyspraxia and the autistic spectrum. Prostaglandins Leukot Essent Fatty Acids, 63(1-2), 1-9.

Ronald, A., Happe, F., Bolton, P., Butcher, L. M., Price, T. S., Wheelwright, S., et al. (2006). Genetic heterogeneity between the three components of the autism spectrum: a twin study. J Am Acad Child Adolesc Psychiatry, 45(6), 691-699.

Saste, M. D., Carver, J. D., Stockard, J. E., Benford, V. J., Chen, L. T., \& Phelps, C. P. (1998). Maternal diet fatty acid composition affects neurodevelopment in rat pups. $J$ Nutr, 128(4), 740-743.

Schachter, H. M., Kourad, K., Merali, Z., Lumb, A., Tran, K., \& Miguelez, M. (2005). Effects of omega-3 fatty acids on mental health. Evid Rep Technol Assess (Summ)(116), 1-11.

Scorza, F. A., Cysneiros, R. M., Arida, R. M., Terra-Bustamante, V. C., de Albuquerque, M., \& Cavalheiro, E. A. (2008). The other side of the coin: Beneficiary effect of omega-3 fatty acids in sudden unexpected death in epilepsy. Epilepsy Behav, 13(2), 279-283.

Sherman, E. M., Slick, D. J., Connolly, M. B., \& Eyrl, K. L. (2007). ADHD, neurological correlates and health-related quality of life in severe pediatric epilepsy. Epilepsia, 48(6), 1083-1091.

Singh, M. (2005). Essential fatty acids, DHA and human brain. Indian J Pediatr, 72(3), 239-242. 
Sinn, N., \& Bryan, J. (2007). Effect of supplementation with polyunsaturated fatty acids and micronutrients on learning and behavior problems associated with child ADHD. J Dev Behav Pediatr, 28(2), 82-91.

Sorgi, P. J., Hallowell, E. M., Hutchins, H. L., \& Sears, B. (2007). Effects of an open-label pilot study with high-dose EPA/DHA concentrates on plasma phospholipids and behavior in children with attention deficit hyperactivity disorder. Nutr J, 6, 16.

Stevens, L. J., Zentall, S. S., Deck, J. L., Abate, M. L., Watkins, B. A., Lipp, S. R., et al. (1995). Essential fatty acid metabolism in boys with attention-deficit hyperactivity disorder. Am $J$ Clin Nutr, 62(4), 761-768.

Tang, A., Wanchoo, S. J., Swann, A. C., \& Dafny, N. (2009). Psychostimulant treatment for ADHD is modulated by prefrontal cortex manipulation. Brain Res Bull, 80(6), 353-358.

Tavriger, R. (1966). Some parental theories about the causes of epilepsy. Epilepsia, 7(4), 339343.

Uauy, R., Hoffman, D. R., Peirano, P., Birch, D. G., \& Birch, E. E. (2001). Essential fatty acids in visual and brain development. Lipids, 36(9), 885-895.

van Goor, S. A., Dijck-Brouwer, D. A., Hadders-Algra, M., Doornbos, B., Erwich, J. J., Schaafsma, A., et al. (2009). Human milk arachidonic acid and docosahexaenoic acid contents increase following supplementation during pregnancy and lactation.

Prostaglandins Leukot Essent Fatty Acids, 80(1), 65-69.

Voigt, R. G., Llorente, A. M., Jensen, C. L., Fraley, J. K., Berretta, M. C., \& Heird, W. C. (2001). A randomized, double-blind, placebo-controlled trial of docosahexaenoic acid supplementation in children with attention-deficit/hyperactivity disorder. J Pediatr, 139(2), 189-196. 
Wainwright, P. E. (2002). Dietary essential fatty acids and brain function: a developmental perspective on mechanisms. Proc Nutr Soc, 61(1), 61-69.

Wing, L., \& Potter, D. (2002). The epidemiology of autistic spectrum disorders: is the prevalence rising? Ment Retard Dev Disabil Res Rev, 8(3), 151-161.

Young, C., Gean, P. W., Chiou, L. C., \& Shen, Y. Z. (2000). Docosahexaenoic acid inhibits synaptic transmission and epileptiform activity in the rat hippocampus. Synapse, 37(2), 90-94.

Yuen, A. W., \& Sander, J. W. (2004). Is omega-3 fatty acid deficiency a factor contributing to refractory seizures and SUDEP? A hypothesis. Seizure, 13(2), 104-107.

Yuen, A. W., Sander, J. W., Fluegel, D., Patsalos, P. N., Bell, G. S., Johnson, T., et al. (2005). Omega-3 fatty acid supplementation in patients with chronic epilepsy: a randomized trial. Epilepsy Behav, 7(2), 253-258. 